\title{
Analysis of 21 autosomal STRs in Saudi Arabia reveals population structure and the influence of consanguinity
}

Yahya M. Khubrani ${ }^{1,2}$, Jon H. Wetton ${ }^{\dagger 1}$, Mark A. Jobling ${ }^{\dagger 1}$

${ }^{1}$ Department of Genetics \& Genome Biology, University of Leicester, University Road, Leicester

${ }^{2}$ Forensic Genetics Laboratory, General Administration of Criminal Evidence, Public Security, Ministry of Interior, Kingdom of Saudi Arabia

+ Correspondence can be addressed to either author:

Dr Jon H. Wetton, Department of Genetics \& Genome Biology, University of Leicester, University Road, Leicester LE1 7RH, UK

Tel.: +44 (0)116 2523377 Fax: +44 (0)116 2523378

Email: jw418@le.ac.uk

Prof Mark A. Jobling, Department of Genetics \& Genome Biology, University of Leicester, University Road, Leicester LE1 7RH, UK

Tel.: +44 (0)1162523427 Fax: +44 (0)1162523378

Email: maj4@le.ac.uk

Running head: Diversity of 21 autosomal STRs in Saudi Arabia 


\section{Abstract}

Variation in the 21 autosomal STRs detected by the GlobalFiler multiplex was investigated in a sample of 523 indigenous male Arabs from five geographic regions of Saudi Arabia. Although allele frequencies for the entire dataset were found to be broadly similar to those determined in previous studies of Saudi citizens, significant differences were found among regions. Heterozygote deficiency was observed at nearly all loci in all regions, probably as a consequence of high levels of consanguineous marriage; in the case of D2S1338, which showed the largest deviation from Hardy-Weinberg equilibrium, the presence of a null allele also played a part. Genetic distances were greatest between the Northern and Southern regions, whilst the West, Central and East appeared most similar to each other, and to previously published surveys. This contrasts with previously described variation among paternal lineages in the same sample-set: Y-chromosome variation was limited within the North/Central/South core compared with the more diverse East and West. Differences between autosomal and Y-chromosomal patterns probably reflect genetic drift on the Y chromosome, exacerbated by prevalent patrilineal descent groups in different regions.

\section{Keywords:}

Saudi Arabia, autosomal STRs, population structure, heterozygosity, consanguinity, GlobalFiler 


\section{Highlights}

- GlobalFiler (21-STR) genotypes reported for 523 Saudi Arabian males

- Significant differences in allele frequencies among five geographical subregions

- Heterozygote deficiency observed at nearly all loci in all regions, likely reflecting history of consanguineous marriage

- Genetic distances greatest between Northern and Southern regions, with West, Central and East most similar to each other

- Autosomal pattern of sub-regional differentiation contrasts with previously described pattern of Y-STR variation in the same sample-set

- Population structure and heterozygote deficiency should be accounted for in forensic interpretation of STR profiles in Saudi Arabia 


\section{Introduction}

The Kingdom of Saudi Arabia (KSA) is located in the southwest corner of Asia at the junction between the three old-world continents of Asia, Africa and Europe. It constitutes the majority of the Arabian Peninsula along with Kuwait, Qatar and the United Arab Emirates to the east, Yemen and Oman to the south, and both Jordan and Iraq to the north. It also faces Egypt, Sudan and Eritrea over the Red Sea to the west, and Iran over the Arabian Gulf to the east. The country is populated by approximately 32.5 million people of whom 20.4 million are Saudi nationals according to Saudi Arabian General Authority for Statistics (last accessed 01/10/2018). Administratively, the country is divided into 13 regions across five geographical areas namely: Central (Riyadh, Al-Qassim), Northern (Northern borders region, Tabuk, Al-Jawf and Hail), Southern (Asir, Jazan, AlBahah and Najran), Eastern (Eastern province) and Western (Makkah and AlMadinah) (Figure 1a).

Several previous studies have investigated autosomal allele frequencies in Saudi Arabia, initially covering just eight STRs among 207 individuals [1], then 190 individuals from the Riyadh region analysed with the 15-STR Identifiler multiplex [2], and 500 individuals from six cities spread across the five regions with the 21 STRs of the GlobalFiler system [3]. Further studies examined variation at 13 (Profiler Plus) and 15 STRs (Identifiler) in Saudis resident in the bordering countries of Dubai [4] and Kuwait [5] respectively. Whilst these studies determined autosomal STR allele frequencies in Saudi nationals, they did not explore population structure within KSA. Analysis of the sample-set described here, which includes approximately equal representation of the five regions of the Kingdom, has previously revealed striking population structure in Y-chromosome variation, with greater diversity in the East and West and relative homogeneity within the North to South central axis of the country [6]. Here we ask whether population structure can also be detected with autosomal markers, and if so whether it presents a similar geographical pattern to that of the patrilineal variation. 


\section{Materials and Methods}

\section{DNA sampling}

Samples were collected from indigenous Saudi Arabian males [6] with continuous paternal ancestry back to their great-grandfather within each of the five geographical subdivisions of the country (Figure 1a; Central $N=115$, Northern $\mathrm{N}=104$, Southern $\mathrm{N}=103$, Eastern $\mathrm{N}=88$, and Western $\mathrm{N}=113$ ) ensuring that all donors were separated by at least three generations. Ethical review for recruitment and analysis was provided by the Saudi General Administration for Forensic Evidence and the University of Leicester Research Ethics Committee. Informed consent was provided by all participants.

\section{DNA Extraction and Quantification}

DNA from FTA blood-spot samples (Whatman, UK) was extracted, purified and quantified as previously described [6].

\section{DNA amplification and fragment detection}

The GlobalFiler@ PCR amplification kit was used to generate profiles based on 21 autosomal STRs: D3S1358, vWA, D16S539, CSF1P0, TP0X, D8S1179, D21S11, D18S51, D2S441, D19S433, TH01, FGA, D22S1045, D5S818, D13S317, D7S820, SE33, D10S1248, D1S1656, D12S391 and D2S1338, along with three additional markers used in sex determination (DYS391, Y indel and Amelogenin). Amplification was performed on a Veriti PCR machine (Thermo Fisher Scientific) and fragment detection on an ABI3500 Genetic Analyzer (Thermo Fisher Scientific) in accordance with the manufacturer's recommended protocols. GeneMapper IDX software V1.4 was used for allele calling and interpretation.

This work followed the guidelines of FSI:Genetics for publication of population genetic data [7-9] and for allele nomenclature [10]. The dataset has been QC checked via STRidER [11], with the dataset reference STR000119. 


\section{Forensic and statistical analysis}

PowerStats v1.2 software (Promega Corporation, Madison, WI, USA) [12] was used to calculate allele frequencies, Random Match Probability (PM), Power of Discrimination (PD), Power of Exclusion (PE), Typical Paternity Index (TPI), observed homozygosity and observed heterozygosity. Arlequin v 3.5 [13] was used to test Hardy-Weinberg equilibrium, calculate expected heterozygosity, perform AMOVA to investigate genetic diversity within and between the five geographical regions, and undertake population differentiation tests for KSA and neighbouring countries. FIS $_{\text {IS }}$ was calculated using FSTAT [14]. Average pairwise FST values for 13 loci (CSF1P0, D13S317, D16S539, D18S51, D21S11, D3S1358, D5S818, D7S820, D8S1179, FGA, TH01, TPOX, vWA) shared with other studies of Saudi Arabians and neighbouring populations were used to generate multidimensional scaling (MDS) plots using the (MASS) package [15] in the R library.

\section{Results}

\section{1- Data descriptions and forensic statistics}

The 21 autosomal STRs targeted by the GlobalFiler kit were amplified from 523 Saudi Arabian males. Table 1 presents allele frequency data and forensic statistics for the whole KSA dataset; these measures are also provided for each of the five geographical subdivisions (Figure 1a) in Table S1.

The least variable loci are TPOX and D22S1045, each with seven allelic variants in the KSA dataset, and the most variable locus was SE33 with 42 alleles. These respective loci had the lowest and highest Probabilities of Discrimination 0.820 , 0.837 and 0.993 respectively), and conversely the highest and lowest major allele frequencies: TPOX allele 8 had a frequency of 0.552 in the total KSA dataset, being most frequent in the North (0.596) and rarest in the Central region (0.517), whilst SE33 allele 18, the most common variant at that locus, was detected at a frequency of just 0.107 in the KSA dataset overall. The combined 
power of discrimination (PD) and the combined power of exclusion (PE) for all loci in the KSA data are 0.9999999999999999999999999705 (PI = $2.95 \times 10^{-26}$ ) and 0.9999999563 , respectively.

\section{2- Rare variants, off-ladder and null alleles}

Thirty-five alleles were each observed only once in the entire dataset, and have been designated "rare" among Saudis. Of these, 13 were also globally uncommon, and were among the 26 off-ladder alleles recorded at these loci: D3S1358 (15.2 [ $\mathrm{N}_{\mathrm{obs}}=1$ ] and 16.2 [1]), D16S539 (13.3 [1]), D18S51 (15.2 [3], 16.2 [6], 17.2 [2]), D2S441 (13.3 [1]), FGA (21.2 [1], 22.2 [2], 22.3 [2], 23.2 [2]), SE33 (7.3 [5], 10 [1], 11.2 [2], 13.2 [1], 13.3 [1], 22 [2], 24 [1], 31 [1], 33 [5], 34 [5], 36.2 [1]), D1S1656 (8 [1],18 [3],19 [1]) and D12S391 (18.3 [4]). All 26 offladder alleles have been described previously in STRBase (http://strbase.nist.gov/index.htm) [16]. No peak was detected at D2S1338 in one individual, despite good signal strength at all other loci, and despite retyping the sample twice. We assume this individual is a null homozygote at this locus and have excluded him from further analyses, as recommended [11].

\section{3- Genetic Structure}

All but one locus showed a deficiency of heterozygotes against expectation in the whole KSA dataset, and this was also apparent within all five regions for between 16 and 20 loci (Table 2 for KSA, and Table S2 for each region). The deviation from Hardy-Weinberg equilibrium was significant following Bonferroni correction ( $\mathrm{P} \leq \mathrm{0.00001})$ for D2S1338 and vWA. Heterozygote deficiency was also evident from AMOVA analysis, with an inbreeding coefficient ( $F_{I S}$ ) of 0.0476 representing $4.71 \%$ of variation for the KSA dataset, whilst $\mathrm{F}_{S T}$ was 0.0021 . F $_{\text {ST }}$ values between the five regions (Table 3) show that the greatest differentiation is between the North and South, with West, Central and East being less differentiated, and the East region being most similar to the other regions, as reflected in an MDS plot (Figure 1b).

Whilst the entire KSA dataset broadly clustered with the previously published Saudi datasets in MDS analysis (Figure 1c), division according to sub-regional 
origin showed that the North and South sub-regions were also the most differentiated from most other nearby populations (Figure 1d). The results of per-locus population differentiation tests between the KSA and regional datasets, previously published Saudi datasets and neighbouring countries are presented in Table S3. 


\section{Discussion}

In our analysis of diversity at the 21 autosomal STR loci of the GlobalFiler multiplex we found no previously unreported alleles among 523 indigenous Saudi Arabian individuals and no evidence of genetic differentiation between the combined KSA dataset and previously published Saudi autosomal datasets, through exact tests of allele frequency. In common with previous studies [1-3, 5], a tendency towards heterozygote deficiency was observed affecting almost all loci, although only two retained significance following Bonferroni correction. This observation, and the elevated $\mathrm{F}_{\text {IS }}$ values, are likely to reflect historical marriage practices in KSA in which partners are usually from within the same tribal group, and rates of first-cousin marriages are high, at around 30\% [17]. One of the two loci that showed significant deviation from Hardy-Weinberg equilibrium due to heterozygote deficiency (D2S1338) also showed evidence of the presence of a null allele, identified through a null homozygote. We assume that the combined effect of consanguinity [17] and presence of null alleles in the heterozygous state produced the apparent excess of CE length "homozygotes" (P $=0.00001)$. It is unclear whether both factors, or only inbreeding, contributed to the significant but slightly weaker distortion observed at vWA $(\mathrm{P}=0.00006)$.

Interestingly, a null has previously been reported at D2S1338 with the Identifiler multiplex, which could be weakly detected as a visible allele with the NGM kit [18]. Identifiler, NGM and GlobalFiler are all Thermo Fisher multiplexes and share exactly the same primers at this locus (Matt Phipps, ThermoFisher personal communication). The same SNP (rs567937457) which lies 174bp downstream of the repeat array was also identified by NIST as causing discordance between Identifiler and Promega PP18 kits (http://strbase.nist.gov/pub_pres/NIST-Update-EDNAP-Apr2011.pdf). It has been suggested that the discrepancy between Identifiler and NGM is related to the longer annealing/extension time (3 min vs. $1 \mathrm{~min}$ ) in the latter, which may permit amplification from the poorly matched primer [18]; however, in our homozygous null individual, extending the annealing/extension time to 3 minutes did not yield a detectable peak. Subsequent amplification with the 
MiniFiler kit which has a 183bp shorter amplicon also yielded no result. The positions of the MiniFiler primers are proprietary information but can encompass at most $60 \mathrm{bp}$ of flanking region which must exclude the aforementioned SNP as the cause, and would imply either a substantial deletion affecting both downstream primers or a polymorphism upstream of the repeat, where the primer is already close to the repeat array. Unfortunately we are unable to explore the nature of this polymorphism any further due to the limitations of the DNA donor consent.

This study is the first to specifically address the question of sub-structure within the indigenous Saudi Arabian population using autosomal STR markers. Our population sample, which has approximately equal representation of the five geographic regions of the Kingdom, has previously been shown to display striking differentiation in Y-chromosomal haplogroup (Y-SNP) and haplotype (YSTR, Yfiler Plus) distributions [6], suggesting that the country is genetically substructured at least with respect to male-specific markers.

A different picture emerged when we explored the genetic diversity of the geographic sub-regions with GlobalFiler. Significantly different pairwise FST $_{\text {T }}$ values were noted between the South, North and Central/West regions, with the East appearing intermediate, and not significantly differentiated from any other region (Figure 1b). The combined KSA dataset is virtually indistinguishable from sets of Saudi donors sampled in Dubai and Kuwait $[4,5]$ and the previous multicity GlobalFiler survey within Saudi Arabia [3], but somewhat different from the Identifiler dataset derived from bone marrow donors at a Riyadh hospital [2], which is an outlier (Figure 1c). However, after division of our dataset into the five regions (Figure 1d) we see that sample-sets from the previously published population surveys now cluster most closely with the Eastern region. Also, the North and South are the most differentiated from each other and from the other regions, with the exception of the bone marrow donor set [2], which shows similarity with the South.

Autosomal differentiation between North and South may be a 
consequence of historically limited migration between these regions. Migrants are now primarily attracted by the oil industry in the East, the holy cities of the West and the capital in the Central region [19]. Prior to the establishment of the Kingdom of Saudi Arabia the interior was sparsely populated by sedentary farmers or nomads who moved within their tribal areas, reflected by some of the current administrative boundaries. The rapid growth of the Saudi population, stimulated by the discovery of oil, has involved movement into the cities from the surrounding areas, but the rural populations have not generally moved between regions. Our regional patrilineal criterion for inclusion in the dataset [6] meant that the results of Y-chromosome tests reflect the historic boundaries of tribal groups, and these are strongest and most stable in the North and Central regions. By contrast, the East and West received the greatest inward migration in recent centuries, as they were outward facing and the destinations of traders and pilgrims. The autosomal results also reflect the movement of women, who are likely to move to their husband's home. We applied no restriction on the origin of the maternal line, and as a consequence a different pattern reflecting more recent migration patterns is unsurprising.

\section{Conclusion}

This study is in concordance with the high discrimination power of GlobalFiler in the Saudi population, which makes it suitable for the purposes of forensic DNA identification and paternity testing; the allele frequencies derived in this study can be utilised by Saudi forensic providers for DNA interpretation purposes. Whilst the observed allele frequency variation between regions will have limited influence on interpretation issues, the high level of consanguinity and presence of null alleles should be taken into account.

\section{Acknowledgments}

YMK acknowledges support from the Saudi Arabian Ministry of Interior, and a PhD studentship grant from the Saudi Arabian Cultural Bureau, London. We 
thank members of the Forensic Genetics Laboratory, General Administration of Criminal Evidence, Riyadh, for assistance, as well as Jon Kyte and Thermo Fisher for their support.

\section{Conflicts of interest}

None 


\section{References}

[1] S. Sinha, M. Amjad, C. Rogers, J.E. Hamby, U.A. Tahir, K. Balamurugan, N.A. alKubaidan, A.R. Choudhry, B. Budowle, M.A. Tahir, Typing of eight short tandem repeat (STR) loci in a Saudi Arabian population, Forensic Sci Int 104 (1999) 1436.

[2] A.E. Osman, H. Alsafar, G.K. Tay, J.B.J.M. Theyab, M. Mubasher, N.E.-E. Sheikh, H. AlHarthi, M.H. Crawford, G. Gehad El Ghazali, Autosomal short tandem repeat (STR) variation based on 15 loci in a population from the Central Region (Riyadh Province) of Saudi Arabia, J. Forensic Res. 6 (2015) 1000267.

[3] H.M. Alsafiah, W.H. Goodwin, S. Hadi, M.A. Alshaikhi, P.P. Wepeba, Population genetic data for 21 autosomal STR loci for the Saudi Arabian population using the GlobalFiler@ PCR amplification kit, Forensic Sci Int Genet 31 (2017) e59e61.

[4] F. Alshamali, A.Q. Alkhayat, B. Budowle, N.D. Watson, STR population diversity in nine ethnic populations living in Dubai, Forensic Sci Int 152 (2005) 267-79.

[5] M. Al-Enizi, J. Ge, S. Ismael, H. Al-Enezi, A. Al-Awadhi, W. Al-Duaij, B. Al-Saleh, Z. Ghulloom, B. Budowle, Population genetic analyses of 15 STR loci from seven forensically-relevant populations residing in the state of Kuwait, Forensic Sci Int Genet 7 (2013) e106-7.

[6] Y.M. Khubrani, J.H. Wetton, M.A. Jobling, Extensive geographical and social structure in the paternal lineages of Saudi Arabia revealed by analysis of $27 \mathrm{Y}$ STRs, Forensic Sci. Int. Genet. 33 (2018) 98-105.

[7] A. Carracedo, J.M. Butler, L. Gusmao, W. Parson, L. Roewer, P.M. Schneider, Publication of population data for forensic purposes, Forensic Sci Int Genet 4 (2010) 145-7.

[8] A. Carracedo, J.M. Butler, L. Gusmao, A. Linacre, W. Parson, L. Roewer, P.M. Schneider, New guidelines for the publication of genetic population data, Forensic Sci Int Genet 7 (2013) 217-20.

[9] L. Gusmao, J.M. Butler, A. Linacre, W. Parson, L. Roewer, P.M. Schneider, A. Carracedo, Revised guidelines for the publication of genetic population data, Forensic Sci Int Genet 30 (2017) 160-163.

[10] P.M. Schneider, Scientific standards for studies in forensic genetics, Forensic Sci Int 165 (2007) 238-43.

[11] M. Bodner, I. Bastisch, J.M. Butler, R. Fimmers, P. Gill, L. Gusmao, N. Morling, C. Phillips, M. Prinz, P.M. Schneider, W. Parson, Recommendations of the DNA Commission of the International Society for Forensic Genetics (ISFG) on quality control of autosomal Short Tandem Repeat allele frequency databasing (STRidER), Forensic Sci Int Genet 24 (2016) 97-102.

[12] A. Tereba, Tools for Analysis of Population Statistics, Profiles in DNA 3 (1999) 14-16; Available from

http://www.promega.com/geneticidtools/powerstats.

[13] L. Excoffier, H.E. Lischer, Arlequin suite ver 3.5: a new series of programs to perform population genetics analyses under Linux and Windows, Mol. Ecol. Resour. 10 (2010) 564-7.

[14] J. Goudet, FSTAT (Version 1.2): A Computer Program to Calculate FStatistics, J. Hered. 86 (1995) 485-486.

[15] W.N. Venables, B.D. Ripley, Modern Applied Statistics with S. Fourth Edition, 
Springer, New York, 2002.

[16] C.M. Ruitberg, D.J. Reeder, J.M. Butler, STRBase: a short tandem repeat DNA database for the human identity testing community, Nucleic Acids Res. 29 (2001) 320-322.

[17] L. Al-Gazali, H. Hamamy, S. Al-Arrayad, Genetic disorders in the Arab world, BMJ 333 (2006) 831-4.

[18] A.A. Westen, T. Kraaijenbrink, E.A. Robles de Medina, J. Harteveld, P. Willemse, S.B. Zuniga, K.J. van der Gaag, N.E. Weiler, J. Warnaar, M. Kayser, T.

Sijen, P. de Knijff, Comparing six commercial autosomal STR kits in a large Dutch population sample, Forensic Sci Int Genet 10 (2014) 55-63.

[19] M. Al-Rasheed, A History of Saudi Arabia, Cambridge University Press, Cambridge, 2010.

[20] A.S. Abuidrees, M.J. Ishaq, C.-E. Pu, N.A. Alhamad, H.A. Alnafea, A.M.

Almehaizea, A Globally used 15 short tandem repeats (STR) loci in forensic human identification, with their allele frequencies and statistical values in the population of Bahrain, Arab Gulf J. Sci. Res. 32 (2014) 177-182.

[21] G.A. Omran, G.N. Rutty, M.A. Jobling, Genetic variation of 15 autosomal STR loci in Upper (Southern) Egyptians, Forensic Sci Int Genet 3 (2009) e39-44. [22] A. Hedjazi, A. Nikbakht, M. Hosseini, A. Hoseinzadeh, S.M. Hosseini, Allele frequencies for 15 autosomal STR loci in Fars province population, southwest of Iran, Leg Med (Tokyo) 15 (2013) 226-8.

[23] M.M. Farhan, S. Hadi, A. Iyengar, W. Goodwin, Population genetic data for 20 autosomal STR loci in an Iraqi Arab population: Application to the identification of human remains, Forensic Sci Int Genet 25 (2016) e10-e11.

[24] L.N. Al-Eitan, R.R. Tubaishat, Evaluation of forensic genetic efficiency parameters of 22 autosomal STR markers (PowerPlex® Fusion system) in a population sample of Arab descent from Jordan, Aus. J. Forensic Sci. 50 (2016) 97-109.

[25] A.M. Perez-Miranda, M.A. Alfonso-Sanchez, J.A. Pena, R.J. Herrera, Qatari DNA variation at a crossroad of human migrations, Hum Hered 61 (2006) 67-79. [26] R.J. Jones, W.A. Tayyare, G.K. Tay, H. Alsafar, W.H. Goodwin, Population data for 21 autosomal short tandem repeat markers in the Arabic population of the United Arab Emirates, Forensic Sci Int Genet 28 (2017) e41-e42.

[27] O. Ali Alhmoudi, R.J. Jones, G.K. Tay, H. Alsafar, S. Hadi, Population genetics data for 21 autosomal STR loci for United Arab Emirates (UAE) population using next generation multiplex STR kit, Forensic Sci Int Genet 19 (2015) 190-191. 


\section{Figure legends}

Figure 1: Map of sample locations, and multidimensional scaling (MDS) plots based on pairwise $F_{S T}$ values derived from autosomal STR data.

a) Map of Saudi Arabia, showing location of the five geographical sub-regions; b) MDS plot comparing the five KSA sub-regions; c) MDS comparison of combined KSA dataset (encircled K) compared to previously published Saudi Arabian datasets and neighbouring countries; d) Five KSA sub-regions (encircled N northern; S - southern; E - eastern; W - western; C- central) compared to previously published Saudi Arabian datasets and neighbouring countries. Comparative data sources and population abbreviations are as follows: $\mathrm{BAH}$ Bahrain [20], EGY1, EGY2 - Egypt, respectively [5, 21]; IRN1, IRN2 - Iran, respectively [5, 22]; IRQ1, IRQ2 - Iraq, respectively [5, 23]; JOR - Jordan [24]; KUW - Kuwait [5]; OMN - Oman [4]; QAT - Qatar [25]; SA1, SA2, SA3, SA4 - Saudi Arabia, respectively [2-5]; UAE1, UAE2 - United Arab Emirates, respectively [26, 27]; YEM - Yemen [4]. 


\section{Tables}

\section{Main text tables:}

Table 1: KSA allele frequencies and forensic statistics.

MP: Random Match Probability, PE: Power of Exclusion, O-Het: observed heterozygosity, E-Het: expected heterozygosity, HWE p-value: probability of deviation from Hardy Weinberg equilibrium, $\mathrm{F}_{I S} \mathrm{p}$-value: significance of $\mathrm{F}_{\text {IS. }}$. 


\begin{tabular}{|c|c|c|c|c|c|c|c|c|c|c|c|c|c|c|c|c|c|c|c|c|c|}
\hline Allele & D3S1358 & vWA & D16S539 & CSF1PO & TPOX & D8S1179 & $\mathrm{D} 21 \mathrm{~S} 11$ & D18S51 & D2S441 & D19S433 & $\mathrm{TH} 01$ & FGA & D22S1045 & D5S818 & D13S317 & D7S820 & SE33 & D10S1248 & D1S1656 & D12S391 & D2S1338 \\
\hline 4 & & & & & & & & & & & 0.001 & & & & & & & & & & \\
\hline 6 & & & & & 0.002 & & & & & & 0.339 & & & & & & & & & & \\
\hline 6.3 & & & & & & & & & & & & & & & & & 0.005 & & & & \\
\hline 7 & & & & 0.001 & 0.001 & & & & & & 0.162 & & & & 0.001 & 0.012 & & & & & \\
\hline 7.3 & & & & & & & & & & & & & & & & & 0.005 & & & & \\
\hline 8 & & & 0.031 & 0.005 & 0.552 & 0.002 & & & & & 0.110 & & & 0.011 & 0.105 & 0.165 & & & 0.001 & & \\
\hline 9 & & & 0.113 & 0.011 & 0.163 & 0.007 & & & 0.011 & & 0.282 & & & 0.076 & 0.033 & 0.111 & 0.004 & 0.018 & & & \\
\hline 9.3 & & & & & & & & & & & 0.096 & & & & & & & & & & \\
\hline 10 & & & 0.128 & 0.304 & 0.113 & 0.038 & & 0.001 & 0.124 & 0.001 & 0.010 & & 0.003 & 0.123 & 0.062 & 0.361 & 0.001 & & 0.002 & & \\
\hline 11 & & & 0.359 & 0.294 & 0.157 & 0.137 & & 0.021 & 0.358 & 0.011 & 0.001 & & 0.107 & 0.262 & 0.255 & 0.183 & 0.001 & 0.009 & 0.042 & & \\
\hline 11.2 & & & & & & & & & & & & & & & & & 0.002 & & & & \\
\hline 11.3 & & & & & & & & & 0.077 & & & & & & & & & & & & \\
\hline 12 & & & 0.212 & 0.345 & 0.012 & 0.188 & & 0.144 & 0.080 & 0.095 & & & 0.011 & 0.346 & 0.341 & 0.146 & 0.006 & 0.037 & 0.131 & & \\
\hline 12.2 & & & & & & & & & & 0.009 & & & & & & & & & & & \\
\hline 13 & 0.007 & 0.002 & 0.137 & 0.033 & & 0.185 & & 0.210 & 0.010 & 0.168 & & & & 0.171 & 0.147 & 0.020 & 0.015 & 0.167 & 0.099 & & \\
\hline 13.2 & & & & & & & & & & 0.046 & & & & & & & 0.001 & & & & \\
\hline 13.3 & & & 0.001 & & & & & & 0.001 & & & & & & & & 0.001 & & & & \\
\hline 14 & 0.060 & 0.029 & 0.017 & 0.006 & & 0.192 & & 0.122 & 0.300 & 0.209 & & & 0.058 & 0.011 & 0.054 & 0.001 & 0.052 & 0.362 & 0.125 & 0.002 & 0.004 \\
\hline 14.2 & & & & & & & & & & 0.048 & & & & & & & & & & & \\
\hline 15 & 0.239 & 0.157 & 0.002 & & & 0.201 & & 0.119 & 0.033 & 0.141 & & & 0.508 & 0.001 & 0.001 & & 0.030 & 0.257 & 0.136 & 0.025 & \\
\hline 15.2 & 0.001 & & & & & & & 0.003 & & 0.126 & & & & & & & & & & & \\
\hline 15.3 & & & & & & & & & & & & & & & & & & & 0.033 & & \\
\hline 16 & 0.314 & 0.282 & & & & 0.043 & & 0.118 & 0.006 & 0.074 & & & 0.258 & & & & 0.058 & 0.105 & 0.251 & 0.011 & 0.077 \\
\hline 16.2 & 0.001 & & & & & & & 0.006 & & 0.053 & & & & & & & & & & & \\
\hline 16.3 & & & & & & & & & & & & & & & & & & & 0.054 & & \\
\hline 17 & 0.267 & 0.272 & & & & 0.008 & & 0.094 & & 0.007 & & & 0.054 & & & & 0.075 & 0.043 & 0.074 & 0.110 & 0.225 \\
\hline 17.2 & & & & & & & & 0.002 & & 0.012 & & & & & & & & & & & \\
\hline 17.3 & & & & & & & & & & & & & & & & & & & 0.022 & & \\
\hline 18 & 0.105 & 0.179 & & & & & & 0.073 & & & & 0.007 & & & & & 0.107 & 0.001 & 0.003 & 0.149 & 0.098 \\
\hline 18.3 & & & & & & & & & & & & & & & & & & & 0.020 & 0.004 & \\
\hline 19 & 0.007 & 0.066 & & & & & & 0.041 & & & & 0.064 & & & & & 0.064 & & 0.001 & 0.133 & 0.127 \\
\hline 19.3 & & & & & & & & & & & & & & & & & & & 0.006 & 0.005 & \\
\hline 20 & & 0.012 & & & & & & 0.026 & & & & 0.070 & & & & & 0.044 & & & 0.100 & 0.239 \\
\hline 20.2 & & & & & & & & & & & & & & & & & 0.007 & & & & \\
\hline 21 & & 0.001 & & & & & & 0.008 & & & & 0.139 & & & & & 0.018 & & & 0.089 & 0.047 \\
\hline 21.2 & & & & & & & & & & & & 0.001 & & & & & 0.076 & & & & \\
\hline 22 & & & & & & & & 0.009 & & & & 0.122 & & & & & 0.002 & & & 0.123 & 0.014 \\
\hline 22.2 & & & & & & & & & & & & 0.002 & & & & & 0.018 & & & & \\
\hline 22.3 & & & & & & & & & & & & 0.002 & & & & & & & & & \\
\hline 23 & & & & & & & & 0.004 & & & & 0.185 & & & & & & & & 0.140 & 0.068 \\
\hline 23.2 & & & & & & & & & & & & 0.002 & & & & & 0.020 & & & & \\
\hline 24 & & & & & & & & 0.001 & & & & 0.226 & & & & & 0.001 & & & 0.057 & 0.064 \\
\hline
\end{tabular}




\begin{tabular}{|c|c|c|c|c|c|}
\hline 25 & & 0.112 & & 0.041 & 0.030 \\
\hline 25.2 & & & 0.027 & & \\
\hline 26 & & 0.052 & & 0.007 & 0.006 \\
\hline 26.2 & & & 0.050 & & \\
\hline 27 & 0.011 & 0.008 & & 0.004 & 0.001 \\
\hline 27.2 & & & 0.050 & & \\
\hline 28 & 0.131 & 0.006 & & & \\
\hline 28.2 & & & 0.042 & & \\
\hline 29 & 0.270 & 0.003 & & & \\
\hline 29.2 & & & 0.036 & & \\
\hline 30 & 0.256 & 0.001 & & & \\
\hline 30.2 & 0.011 & & 0.043 & & \\
\hline 31 & 0.046 & & 0.001 & & \\
\hline 31.2 & 0.103 & 0.001 & 0.049 & & \\
\hline 32 & 0.007 & & & & \\
\hline 32.2 & 0.112 & & 0.038 & & \\
\hline 33 & & & 0.005 & & \\
\hline 33.2 & 0.039 & & 0.007 & & \\
\hline 34 & & & 0.005 & & \\
\hline 34.2 & 0.007 & & 0.005 & & \\
\hline 35 & 0.005 & & 0.002 & & \\
\hline 35.2 & 0.001 & & 0.001 & & \\
\hline 36 & 0.001 & & & & \\
\hline 36.2 & & & 0.001 & & \\
\hline 37 & 0.001 & & 0.001 & & \\
\hline
\end{tabular}

\begin{tabular}{|c|c|c|c|c|c|c|c|c|c|c|c|c|c|c|c|c|c|c|c|c|c|}
\hline & D3S1358 & vWA & D16S539 & CSF1PO & TPOX & D8S1179 & D21S11 & D18S51 & D2S441 & D19S433 & ТН01 & FGA & D22S1045 & D5S818 & D13S317 & D7S820 & SE33 & D10S1248 & D1S1656 & D12S391 & D2S1338 \\
\hline MP & 0.097 & 0.078 & 0.081 & 0.147 & 0.180 & 0.052 & 0.056 & 0.029 & 0.100 & 0.030 & 0.097 & 0.038 & 0.163 & 0.092 & 0.081 & 0.081 & 0.007 & 0.096 & 0.032 & 0.024 & 0.040 \\
\hline $\mathrm{PE}$ & 0.474 & 0.471 & 0.490 & 0.385 & 0.287 & 0.644 & 0.556 & 0.685 & 0.443 & 0.656 & 0.486 & 0.659 & 0.291 & 0.461 & 0.452 & 0.490 & 0.832 & 0.566 & 0.634 & 0.659 & 0.570 \\
\hline O-Het & 0.729 & 0.727 & 0.738 & 0.671 & 0.597 & 0.824 & 0.778 & 0.845 & 0.709 & 0.830 & 0.736 & 0.832 & 0.600 & 0.721 & 0.715 & 0.738 & 0.918 & 0.782 & 0.818 & 0.832 & 0.784 \\
\hline E-Het & 0.759 & 0.785 & 0.777 & 0.701 & 0.632 & 0.832 & 0.818 & 0.876 & 0.754 & 0.871 & 0.759 & 0.857 & 0.658 & 0.762 & 0.779 & 0.775 & 0.948 & 0.761 & 0.864 & 0.890 & 0.850 \\
\hline P-value & 0.264 & $<0.0001$ & 0.019 & 0.141 & 0.444 & 0.944 & 0.637 & 0.226 & 0.004 & 0.874 & 0.092 & 0.130 & 0.029 & 0.158 & 0.020 & 0.374 & 0.053 & 0.364 & 0.559 & 0.068 & $<0.0001$ \\
\hline Alleles & 9 & 9 & 9 & 8 & 7 & 10 & 15 & 18 & 10 & 14 & 8 & 18 & 7 & 8 & 9 & 8 & 42 & 9 & 16 & 16 & 14 \\
\hline $\mathrm{F}_{\text {IS }}$ & 0.037 & 0.072 & 0.050 & 0.043 & 0.058 & 0.010 & 0.047 & 0.032 & 0.064 & 0.045 & 0.027 & 0.027 & 0.086 & 0.049 & 0.078 & 0.049 & 0.032 & -0.036 & 0.055 & 0.065 & 0.076 \\
\hline P-value & 0.072 & 0.001 & 0.014 & 0.070 & 0.020 & 0.321 & 0.011 & 0.029 & 0.005 & 0.005 & 0.135 & 0.067 & 0.001 & 0.022 & 0.001 & 0.020 & 0.002 & 0.952 & 0.001 & $<0.0001$ & $<0.0001$ \\
\hline
\end{tabular}


Table 2: Pairwise FsT between regional sub-populations.

\begin{tabular}{|l|ccccc|}
\hline Region & $\mathbf{C}$ & $\mathbf{E}$ & $\mathbf{N}$ & $\mathbf{S}$ & $\mathbf{W}$ \\
\hline $\mathbf{C}$ & $*$ & 0.0322 & $\mathbf{0 . 0 0 2 0}$ & $<\mathbf{0 . 0 0 0 1}$ & 0.5664 \\
$\mathbf{E}$ & 0.0017 & $*$ & 0.1006 & 0.1084 & 0.8291 \\
$\mathbf{N}$ & & & & & \\
$\mathbf{S}$ & 0.0028 & 0.0013 & $*$ & $<\mathbf{0 . 0 0 0 1}$ & $\mathbf{0 . 0 0 1 0}$ \\
$\mathbf{W}$ & 0.0041 & 0.0012 & 0.0053 & $*$ & 0.0166 \\
\hline
\end{tabular}

Figures upper-right are p-values, with significant values in bold. 
a
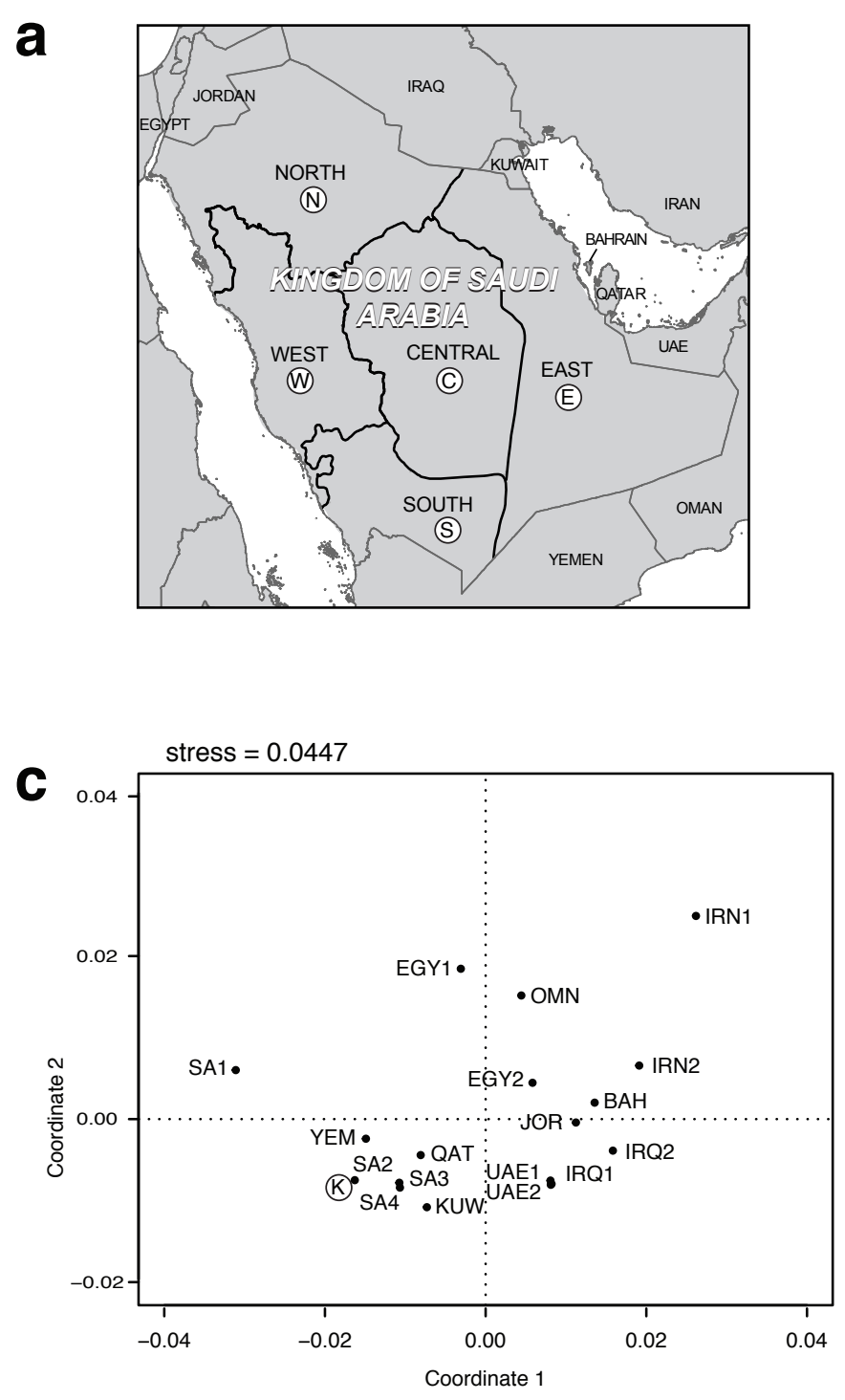
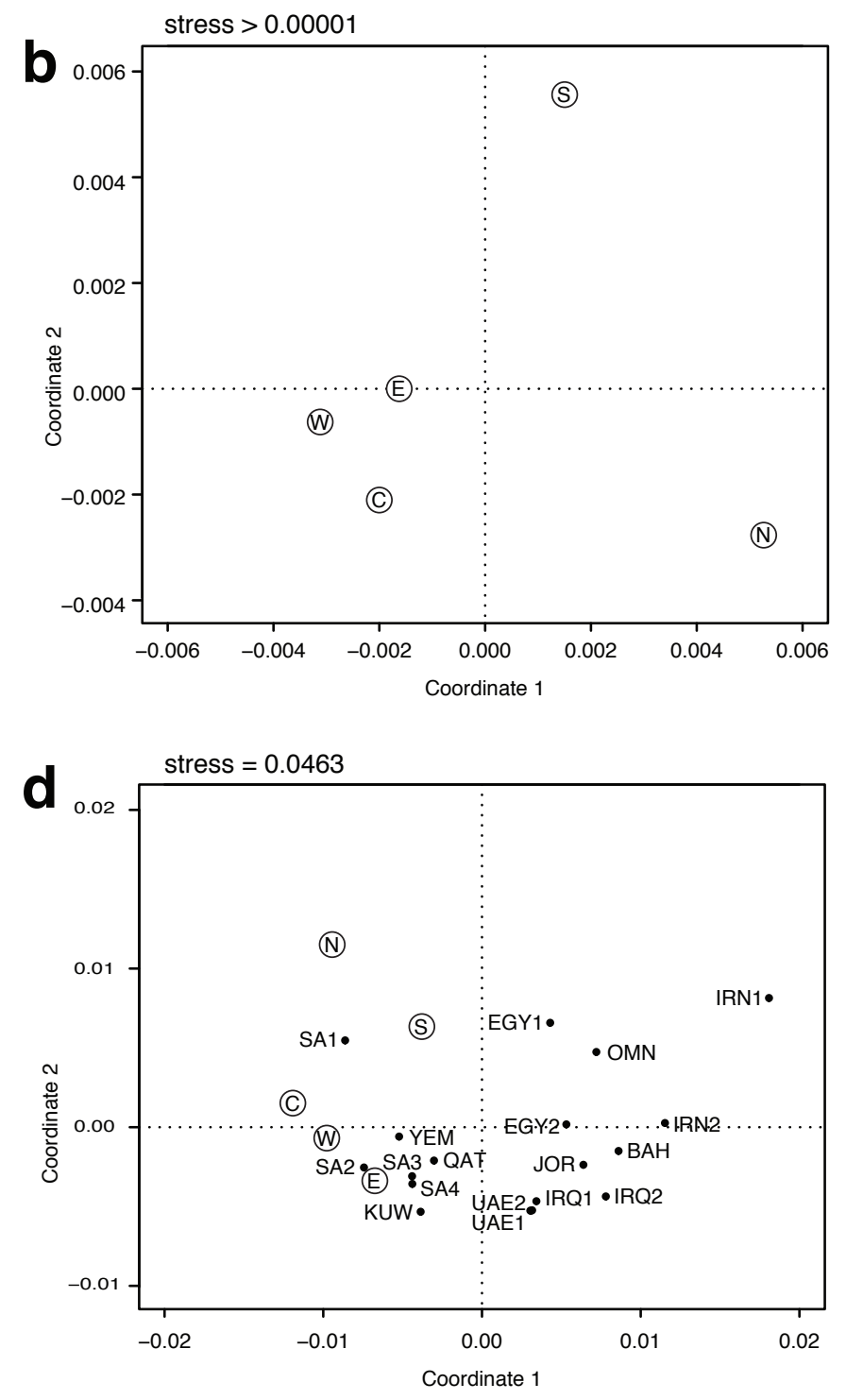

Figure 1. 
Supplementary material, Table S1a. Allele frequencies and forensic statistics (Central region)

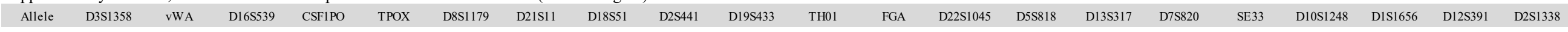

\begin{tabular}{|c|c|c|c|c|c|c|c|c|c|c|c|c|c|c|c|c|c|c|c|c|}
\hline 6 & & & & & & & & & & 0.404 & & & & & & & & & & \\
\hline 6.3 & & & & & & & & & & & & & & & & 0.004 & & & & \\
\hline 7 & & & & & & & & & & 0.135 & & & & 0.004 & 0.004 & & & & & \\
\hline 7.3 & & & & & & & & & & & & & & & & 0.004 & & & & \\
\hline 8 & & & 0.035 & & 0.517 & & & & & 0.096 & & & 0.009 & 0.087 & 0.196 & & & 0.004 & & \\
\hline 9 & & & 0.100 & 0.013 & 0.152 & 0.013 & & 0.009 & & 0.287 & & & 0.065 & 0.026 & 0.096 & & 0.017 & & & \\
\hline 9.3 & & & & & & & & & & 0.074 & & & & & & & & & & \\
\hline 10 & & & 0.078 & 0.317 & 0.135 & 0.022 & & 0.157 & 0.004 & 0.004 & & 0.004 & 0.100 & 0.061 & 0.374 & & & & & \\
\hline 11 & & & 0.391 & 0.261 & 0.174 & 0.157 & 0.022 & 0.365 & 0.004 & & & 0.078 & 0.235 & 0.278 & 0.157 & & & 0.043 & & \\
\hline \multicolumn{21}{|l|}{11.2} \\
\hline 11.3 & & & & & & & & 0.074 & & & & & & & & & & & & \\
\hline 12 & & & 0.257 & 0.374 & 0.022 & 0.196 & 0.174 & 0.057 & 0.100 & & & 0.004 & 0.404 & 0.343 & 0.148 & 0.004 & 0.030 & 0.130 & & \\
\hline 12.2 & & & & & & & & & 0.009 & & & & & & & & & & & \\
\hline 13 & 0.004 & & 0.117 & 0.022 & & 0.157 & 0.217 & 0.013 & 0.130 & & & & 0.178 & 0.157 & 0.026 & 0.013 & 0.126 & 0.130 & & \\
\hline 13.2 & & & & & & & & & 0.030 & & & & & & & & & & & \\
\hline 13.3 & & & 0.004 & & & & & & & & & & & & & 0.004 & & & & \\
\hline 14 & 0.070 & 0.026 & 0.013 & 0.013 & & 0.183 & 0.117 & 0.287 & 0.204 & & & 0.083 & 0.009 & 0.043 & & 0.052 & 0.357 & 0.122 & & 0.004 \\
\hline 14.2 & & & & & & & & & 0.087 & & & & & & & & & & & \\
\hline 15 & 0.261 & 0.130 & 0.004 & & & 0.226 & 0.100 & 0.039 & 0.143 & & & 0.517 & & & & 0.035 & 0.309 & 0.126 & 0.035 & \\
\hline 15.2 & & & & & & & 0.004 & & 0.104 & & & & & & & & & & & \\
\hline 15.3 & & & & & & & & & & & & & & & & & & 0.043 & & \\
\hline 16 & 0.313 & 0.235 & & & & 0.043 & 0.130 & & 0.104 & & & 0.248 & & & & 0.039 & 0.113 & 0.226 & 0.004 & 0.113 \\
\hline 16.2 & & & & & & & 0.013 & & 0.061 & & & & & & & & & & & \\
\hline 16.3 & & & & & & & & & & & & & & & & & & 0.026 & & \\
\hline 17 & 0.213 & 0.317 & & & & 0.004 & 0.087 & & 0.009 & & & 0.065 & & & & 0.083 & 0.048 & 0.100 & 0.083 & 0.217 \\
\hline 17.2 & & & & & & & & & 0.009 & & & & & & & & & & & \\
\hline 17.3 & & & & & & & & & & & & & & & & & & 0.017 & & \\
\hline 18 & 0.130 & 0.200 & & & & & 0.070 & & & & 0.004 & & & & & 0.117 & & 0.009 & 0.100 & 0.091 \\
\hline 18.3 & & & & & & & & & & & & & & & & & & 0.004 & 0.009 & \\
\hline 19 & 0.009 & 0.078 & & & & & 0.030 & & & & 0.043 & & & & & 0.057 & & & 0.143 & 0.126 \\
\hline 19.3 & & & & & & & & & & & & & & & & & & 0.017 & 0.004 & \\
\hline 20 & & 0.013 & & & & & 0.013 & & & & 0.065 & & & & & 0.065 & & & 0.113 & 0.283 \\
\hline 20.2 & & & & & & & & & & & & & & & & 0.009 & & & & \\
\hline 21 & & & & & & & 0.009 & & & & 0.126 & & & & & 0.017 & & & 0.083 & 0.022 \\
\hline
\end{tabular}




\begin{tabular}{|c|c|c|c|c|c|}
\hline 21.2 & & & 0.070 & & \\
\hline 22 & 0.004 & 0.083 & & 0.148 & 0.013 \\
\hline 22.2 & & & 0.026 & & \\
\hline 22.3 & & 0.004 & & & \\
\hline 23 & 0.009 & 0.261 & & 0.170 & 0.052 \\
\hline 23.2 & & 0.004 & 0.022 & & \\
\hline 24 & & 0.226 & 0.004 & 0.057 & 0.061 \\
\hline 24.2 & & & 0.022 & & \\
\hline 25 & & 0.122 & & 0.039 & 0.013 \\
\hline 25.2 & & & 0.026 & & \\
\hline 26 & & 0.043 & & 0.004 & \\
\hline 26.2 & & & 0.043 & & \\
\hline 27 & 0.013 & 0.009 & & 0.009 & 0.004 \\
\hline 27.2 & & & 0.043 & & \\
\hline 28 & 0.096 & 0.004 & & & \\
\hline 28.2 & & & 0.043 & & \\
\hline 29 & 0.257 & 0.004 & & & \\
\hline 29.2 & & & 0.013 & & \\
\hline 30 & 0.270 & & & & \\
\hline 30.2 & 0.004 & & 0.052 & & \\
\hline 31 & 0.043 & & & & \\
\hline 31.2 & 0.152 & & 0.052 & & \\
\hline \multicolumn{6}{|l|}{32} \\
\hline 32.2 & 0.100 & & 0.030 & & \\
\hline 33 & & & 0.009 & & \\
\hline 33.2 & 0.048 & & 0.013 & & \\
\hline \multicolumn{6}{|l|}{34} \\
\hline 34.2 & 0.009 & & 0.013 & & \\
\hline 35 & 0.009 & & 0.009 & & \\
\hline \multicolumn{6}{|l|}{35.2} \\
\hline \multicolumn{6}{|l|}{36} \\
\hline 36.2 & & & 0.004 & & \\
\hline 37 & & & & & \\
\hline
\end{tabular}

\begin{tabular}{|c|c|c|c|c|c|c|c|c|c|c|c|c|c|c|c|c|c|c|c|c|c|}
\hline & D3S1358 & vWA & D16S539 & CSFIPO & TPOX & D8S1179 & D21S11 & D18551 & D2S441 & D19S433 & TH01 & FGA & D22S1045 & D5S818 & D13S317 & D7S820 & SE33 & D10S1248 & D1S1656 & D12S391 & D2S1338 \\
\hline MP & 0.107 & 0.084 & 0.105 & 0.169 & 0.165 & 0.060 & 0.058 & 0.039 & 0.115 & 0.033 & 0.118 & 0.051 & 0.172 & 0.112 & 0.092 & 0.092 & 0.014 & 0.101 & 0.035 & 0.026 & 0.053 \\
\hline PD & 0.893 & 0.916 & 0.895 & 0.831 & 0.835 & 0.940 & 0.942 & 0.961 & 0.885 & 0.967 & 0.882 & 0.949 & 0.828 & 0.888 & 0.908 & 0.908 & 0.986 & 0.899 & 0.965 & 0.974 & 0.947 \\
\hline
\end{tabular}




\begin{tabular}{|c|c|c|c|c|c|c|c|c|c|c|c|c|c|c|c|c|c|c|c|c|c|}
\hline PIC & 0.729 & 0.747 & 0.715 & 0.629 & 0.618 & 0.802 & 0.791 & 0.854 & 0.712 & 0.864 & 0.678 & 0.815 & 0.609 & 0.696 & 0.732 & 0.733 & 0.943 & 0.706 & 0.856 & 0.875 & 0.811 \\
\hline PE & 0.552 & 0.477 & 0.449 & 0.449 & 0.395 & 0.648 & 0.583 & 0.682 & 0.536 & 0.615 & 0.358 & 0.632 & 0.323 & 0.358 & 0.370 & 0.435 & 0.876 & 0.422 & 0.716 & 0.615 & 0.567 \\
\hline TPI & 2.212 & 1.855 & 1.742 & 1.742 & 1.554 & 2.875 & 2.396 & 3.194 & 2.130 & 2.614 & 1.438 & 2.738 & 1.337 & 1.438 & 1.474 & 1.691 & 8.214 & 1.643 & 3.594 & 2.614 & 2.300 \\
\hline Ho & 0.226 & 0.270 & 0.287 & 0.287 & 0.322 & 0.174 & 0.209 & 0.157 & 0.235 & 0.191 & 0.348 & 0.183 & 0.374 & 0.348 & 0.339 & 0.296 & 0.061 & 0.304 & 0.139 & 0.191 & 0.217 \\
\hline $\mathrm{Ht}$ & 0.774 & 0.730 & 0.713 & 0.713 & 0.678 & 0.826 & 0.791 & 0.843 & 0.765 & 0.809 & 0.652 & 0.817 & 0.626 & 0.652 & 0.661 & 0.704 & 0.939 & 0.696 & 0.861 & 0.809 & 0.783 \\
\hline Alleles & 230 & 230 & 230 & 230 & 230 & 230 & 230 & 230 & 230 & 230 & 230 & 230 & 230 & 230 & 230 & 230 & 230 & 230 & 230 & 230 & 230 \\
\hline
\end{tabular}

MP: Random Match Probability, PD: Power of Discrimination, PIC: Polymorphism information content, PE: Power of Exclusion, TPI: Typical Paternity Index, Ho: Homozygosity, Ht: Heterozygosity 
Supplementary material, Table S1b. Allele frequencies and forensic statistics (Eastern region)

\begin{tabular}{|c|c|c|c|c|c|c|c|c|c|c|c|c|c|c|c|c|c|c|c|c|c|}
\hline Allele & D3S1358 & vWA & D16S539 & CSFIPO & TPOX & D8S1179 & D21S11 & D18S51 & D2S441 & D19S433 & TH01 & FGA & D22S1045 & D5S818 & D13S317 & D7S820 & SE 33 & D10S1248 & Dis1656 & D12S391 & D2S1338 \\
\hline \multicolumn{22}{|l|}{4} \\
\hline 6 & & & & & 0.011 & & & & & & 0.335 & & & & & & & & & & \\
\hline 6.3 & & & & & & & & & & & & & & & & & 0.011 & & & & \\
\hline 7 & & & & & & & & & & & 0.153 & & & & & 0.011 & & & & & \\
\hline \multicolumn{22}{|l|}{7.3} \\
\hline 8 & & & 0.034 & 0.023 & 0.585 & 0.006 & & & & & 0.102 & & & 0.017 & 0.119 & 0.153 & & & & & \\
\hline 9 & & & 0.148 & 0.011 & 0.114 & 0.006 & & & 0.011 & & 0.295 & & & 0.068 & 0.028 & 0.114 & 0.006 & & & & \\
\hline 9.3 & & & & & & & & & & & 0.080 & & & & & & & & & & \\
\hline 10 & & & 0.148 & 0.324 & 0.091 & 0.034 & & 0.006 & 0.085 & & 0.034 & & & 0.085 & 0.068 & 0.381 & & & & & \\
\hline 11 & & & 0.341 & 0.261 & 0.193 & 0.102 & & 0.028 & 0.381 & 0.017 & & & 0.142 & 0.335 & 0.273 & 0.170 & & & 0.063 & & \\
\hline \multicolumn{22}{|l|}{11.2} \\
\hline 11.3 & & & & & & & & & 0.080 & & & & & & & & & & & & \\
\hline 12 & & & 0.222 & 0.330 & 0.006 & 0.182 & & 0.119 & 0.080 & 0.091 & & & 0.011 & 0.330 & 0.301 & 0.148 & & 0.063 & 0.136 & & \\
\hline \multicolumn{22}{|l|}{12.2} \\
\hline 13 & & 0.011 & 0.108 & 0.045 & & 0.205 & & 0.176 & 0.006 & 0.182 & & & & 0.153 & 0.159 & 0.023 & 0.017 & 0.210 & 0.085 & & \\
\hline 13.2 & & & & & & & & & & 0.028 & & & & & & & & & & & \\
\hline \multicolumn{22}{|l|}{13.3} \\
\hline 14 & 0.057 & 0.023 & & 0.006 & & 0.205 & & 0.074 & 0.313 & 0.216 & & & 0.063 & 0.006 & 0.051 & & 0.045 & 0.369 & 0.131 & 0.006 & \\
\hline 14.2 & & & & & & & & & & 0.017 & & & & & & & & & & & \\
\hline 15 & 0.239 & 0.193 & & & & 0.210 & & 0.153 & 0.045 & 0.142 & & & 0.517 & 0.006 & & & 0.057 & 0.216 & 0.153 & 0.011 & \\
\hline 15.2 & & & & & & & & & & 0.131 & & & & & & & & & & & \\
\hline 15.3 & & & & & & & & & & & & & & & & & & & 0.028 & & \\
\hline 16 & 0.267 & 0.324 & & & & 0.045 & & 0.131 & & 0.063 & & & 0.199 & & & & 0.063 & 0.102 & 0.210 & 0.023 & 0.034 \\
\hline 16.2 & & & & & & & & 0.006 & & 0.085 & & & & & & & & & & & \\
\hline 16.3 & & & & & & & & & & & & & & & & & & & 0.051 & & \\
\hline 17 & 0.295 & 0.188 & & & & 0.006 & & 0.108 & & 0.017 & & & 0.068 & & & & 0.080 & 0.034 & 0.080 & 0.119 & 0.222 \\
\hline 17.2 & & & & & & & & 0.006 & & 0.011 & & & & & & & & & & & \\
\hline 17.3 & & & & & & & & & & & & & & & & & & & 0.040 & & \\
\hline 18 & 0.136 & 0.165 & & & & & & 0.074 & & & & 0.006 & & & & & 0.068 & 0.006 & & 0.159 & 0.119 \\
\hline 18.3 & & & & & & & & & & & & & & & & & & & 0.023 & & \\
\hline 19 & 0.006 & 0.080 & & & & & & 0.034 & & & & 0.057 & & & & & 0.057 & & & 0.125 & 0.108 \\
\hline 19.3 & & & & & & & & & & & & & & & & & & & & 0.006 & \\
\hline 20 & & 0.017 & & & & & & 0.057 & & & & 0.080 & & & & & 0.034 & & & 0.080 & 0.233 \\
\hline 20.2 & & & & & & & & & & & & & & & & & 0.006 & & & & \\
\hline 21 & & & & & & & & 0.006 & & & & 0.153 & & & & & 0.023 & & & 0.091 & 0.057 \\
\hline 21.2 & & & & & & & & & & & & & & & & & 0.028 & & & & \\
\hline 22 & & & & & & & & 0.017 & & & & 0.125 & & & & & 0.006 & & & 0.125 & \\
\hline
\end{tabular}




\begin{tabular}{|c|c|c|c|c|c|}
\hline 22.2 & & 0.006 & 0.023 & & \\
\hline 22.3 & & 0.006 & & & \\
\hline 23 & & 0.148 & & 0.108 & 0.068 \\
\hline 23.2 & & & 0.023 & & \\
\hline 24 & 0.006 & 0.244 & & 0.091 & 0.097 \\
\hline 24.2 & & & 0.028 & & \\
\hline 25 & & 0.131 & & 0.051 & 0.045 \\
\hline 25.2 & & & 0.051 & & \\
\hline 26 & & 0.034 & & 0.006 & 0.017 \\
\hline 26.2 & & & 0.063 & & \\
\hline 27 & 0.011 & 0.006 & & & \\
\hline 27.2 & & & 0.057 & & \\
\hline 28 & 0.142 & 0.006 & & & \\
\hline 28.2 & & & 0.034 & & \\
\hline 29 & 0.318 & & & & \\
\hline 29.2 & & & 0.080 & & \\
\hline 30 & 0.182 & & & & \\
\hline 30.2 & 0.011 & & 0.045 & & \\
\hline 31 & 0.045 & & & & \\
\hline 31.2 & 0.119 & & 0.051 & & \\
\hline 32 & 0.011 & & & & \\
\hline 32.2 & 0.114 & & 0.040 & & \\
\hline \multicolumn{6}{|l|}{33} \\
\hline 33.2 & 0.034 & & & & \\
\hline \multicolumn{6}{|l|}{34} \\
\hline 34.2 & 0.011 & & & & \\
\hline \multicolumn{6}{|l|}{35} \\
\hline \multicolumn{6}{|l|}{35.2} \\
\hline \multicolumn{6}{|l|}{36} \\
\hline \multicolumn{6}{|l|}{36.2} \\
\hline 37 & & & 0.006 & & \\
\hline
\end{tabular}

\begin{tabular}{|c|c|c|c|c|c|c|c|c|c|c|c|c|c|c|c|c|c|c|c|c|c|}
\hline & D3S1358 & $\mathrm{vWA}$ & D16S539 & CSFIPO & TPOX & D8S1179 & D21S11 & D18S51 & D2S441 & D19S433 & TH01 & FGA & D22S1045 & D5S818 & D13S317 & D7S820 & SE33 & D10S1248 & D1S1656 & D12S391 & D2S1338 \\
\hline MP & 0.099 & 0.084 & 0.081 & 0.132 & 0.209 & 0.057 & 0.061 & 0.033 & 0.126 & 0.042 & 0.107 & 0.044 & 0.157 & 0.109 & 0.079 & 0.095 & 0.015 & 0.115 & 0.038 & 0.028 & 0.046 \\
\hline PD & 0.901 & 0.916 & 0.919 & 0.868 & 0.791 & 0.943 & 0.939 & 0.967 & 0.874 & 0.958 & 0.893 & 0.956 & 0.843 & 0.891 & 0.921 & 0.905 & 0.985 & 0.885 & 0.962 & 0.972 & 0.954 \\
\hline PIC & 0.723 & 0.758 & 0.746 & 0.662 & 0.557 & 0.801 & 0.792 & 0.875 & 0.696 & 0.847 & 0.722 & 0.834 & 0.625 & 0.702 & 0.757 & 0.736 & 0.946 & 0.721 & 0.861 & 0.881 & 0.833 \\
\hline PE & 0.549 & 0.472 & 0.491 & 0.401 & 0.198 & 0.591 & 0.510 & 0.768 & 0.435 & 0.677 & 0.529 & 0.570 & 0.308 & 0.401 & 0.570 & 0.591 & 0.791 & 0.791 & 0.549 & 0.699 & 0.591 \\
\hline TPI & 2.200 & 1.833 & 1.913 & 1.571 & 1.023 & 2.444 & 2.000 & 4.400 & 1.692 & 3.143 & 2.095 & 2.316 & 1.294 & 1.571 & 2.316 & 2.444 & 4.889 & 4.889 & 2.200 & 3.385 & 2.444 \\
\hline Ho & 0.227 & 0.273 & 0.261 & 0.318 & 0.489 & 0.205 & 0.250 & 0.114 & 0.295 & 0.159 & 0.239 & 0.216 & 0.386 & 0.318 & 0.216 & 0.205 & 0.102 & 0.102 & 0.227 & 0.148 & 0.205 \\
\hline
\end{tabular}




\begin{tabular}{|c|c|c|c|c|c|c|c|c|c|c|c|c|c|c|c|c|c|c|c|c|c|}
\hline $\mathrm{Ht}$ & 0.773 & 0.727 & 0.739 & 0.682 & 0.511 & 0.795 & 0.750 & 0.886 & 0.705 & 0.841 & 0.761 & 0.784 & 0.614 & 0.682 & 0.784 & 0.795 & 0.898 & 0.898 & 0.773 & 0.852 & 0.795 \\
\hline Alleles & 176 & 176 & 176 & 176 & 176 & 176 & 176 & 176 & 176 & 176 & 176 & 176 & 176 & 176 & 176 & 176 & 176 & 176 & 176 & 176 & 176 \\
\hline
\end{tabular}

MP: Random Match Probability, PD: Power of Discrimination, PIC: Polymorphism information content, PE: Power of Exclusion, TPI: Typical Paternity Index, Ho: Homozygosity, Ht: Heterozygosity 
Supplementary material, Table S1c. Allele frequencies and forensic statistics (Northern region)

\begin{tabular}{|c|c|c|c|c|c|c|c|c|c|c|c|c|c|c|c|c|c|c|c|c|c|}
\hline Allele & D3S1358 & vWA & D16S539 & CSF1PO & TPOX & D8S1179 & $\mathrm{D} 21 \mathrm{~S} 11$ & D18S51 & D2S441 & D19S433 & TH01 & FGA & D22S1045 & D5S818 & D13S317 & D7S820 & SE33 & D10S1248 & D1S1656 & D12S391 & D2S1338 \\
\hline \multicolumn{22}{|l|}{4} \\
\hline 6 & & & & & & & & & & & 0.313 & & & & & & & & & & \\
\hline 6.3 & & & & & & & & & & & & & & & & & 0.005 & & & & \\
\hline 7 & & & & 0.005 & & & & & & & 0.173 & & & & & 0.010 & & & & & \\
\hline \multicolumn{22}{|l|}{7.3} \\
\hline 8 & & & 0.024 & & 0.596 & & & & & & 0.135 & & & 0.005 & 0.072 & 0.149 & & & & & \\
\hline 9 & & & 0.091 & 0.005 & 0.154 & 0.005 & & & 0.005 & & 0.250 & & & 0.130 & 0.063 & 0.173 & 0.010 & 0.019 & & & \\
\hline 9.3 & & & & & & & & & & & 0.120 & & & & & & & & & & \\
\hline 10 & & & 0.149 & 0.279 & 0.087 & 0.043 & & & 0.139 & & 0.005 & & & 0.154 & 0.077 & 0.337 & & & 0.010 & & \\
\hline 11 & & & 0.361 & 0.298 & 0.149 & 0.154 & & 0.014 & 0.351 & 0.005 & 0.005 & & 0.115 & 0.303 & 0.274 & 0.236 & & 0.014 & 0.053 & & \\
\hline 11.2 & & & & & & & & & & & & & & & & & 0.005 & & & & \\
\hline 11.3 & & & & & & & & & 0.096 & & & & & & & & & & & & \\
\hline 12 & & & 0.212 & 0.380 & 0.014 & 0.183 & & 0.091 & 0.067 & 0.101 & & & 0.014 & 0.240 & 0.269 & 0.087 & 0.010 & 0.014 & 0.111 & & \\
\hline 12.2 & & & & & & & & & & 0.005 & & & & & & & & & & & \\
\hline 13 & & & 0.149 & 0.029 & & 0.173 & & 0.250 & 0.010 & 0.183 & & & & 0.144 & 0.159 & 0.010 & 0.010 & 0.101 & 0.043 & & \\
\hline 13.2 & & & & & & & & & & 0.038 & & & & & & & 0.005 & & & & \\
\hline 13.3 & & & & & & & & & 0.005 & & & & & & & & & & & & \\
\hline 14 & 0.053 & 0.034 & 0.010 & 0.005 & & 0.149 & & 0.178 & 0.288 & 0.245 & & & 0.053 & 0.024 & 0.082 & & 0.058 & 0.365 & 0.149 & 0.005 & \\
\hline 14.2 & & & & & & & & & & 0.038 & & & & & & & & & & & \\
\hline 15 & 0.240 & 0.163 & 0.005 & & & 0.221 & & 0.144 & 0.029 & 0.168 & & & 0.563 & & 0.005 & & 0.024 & 0.313 & 0.154 & 0.005 & \\
\hline 15.2 & 0.005 & & & & & & & & & 0.111 & & & & & & & & & & & \\
\hline 15.3 & & & & & & & & & & & & & & & & & & & 0.019 & & \\
\hline 16 & 0.404 & 0.250 & & & & 0.058 & & 0.115 & 0.010 & 0.048 & & & 0.221 & & & & 0.067 & 0.139 & 0.293 & & 0.087 \\
\hline 16.2 & & & & & & & & 0.005 & & 0.058 & & & & & & & & & & & \\
\hline 16.3 & & & & & & & & & & & & & & & & & & & 0.038 & & \\
\hline 17 & 0.212 & 0.317 & & & & 0.014 & & 0.082 & & & & & 0.034 & & & & 0.072 & 0.034 & 0.082 & 0.082 & 0.216 \\
\hline \multicolumn{22}{|l|}{17.2} \\
\hline 17.3 & & & & & & & & & & & & & & & & & & & 0.010 & & \\
\hline 18 & 0.082 & 0.159 & & & & & & 0.067 & & & & 0.005 & & & & & 0.111 & & 0.005 & 0.207 & 0.091 \\
\hline 18.3 & & & & & & & & & & & & & & & & & & & 0.024 & & \\
\hline 19 & 0.005 & 0.072 & & & & & & 0.010 & & & & 0.077 & & & & & 0.077 & & 0.005 & 0.163 & 0.101 \\
\hline 19.3 & & & & & & & & & & & & & & & & & & & 0.005 & 0.005 & \\
\hline 20 & & 0.005 & & & & & & 0.024 & & & & 0.043 & & & & & 0.029 & & & 0.120 & 0.245 \\
\hline \multicolumn{22}{|l|}{20.2} \\
\hline 21 & & & & & & & & 0.010 & & & & 0.125 & & & & & 0.010 & & & 0.096 & 0.038 \\
\hline 21.2 & & & & & & & & & & & & & & & & & 0.058 & & & & \\
\hline 22 & & & & & & & & 0.005 & & & & 0.159 & & & & & 0.005 & & & 0.091 & \\
\hline
\end{tabular}




\begin{tabular}{|c|c|c|c|c|c|}
\hline 22.2 & & & 0.019 & & \\
\hline \multicolumn{6}{|l|}{22.3} \\
\hline 23 & 0.005 & 0.154 & & 0.111 & 0.091 \\
\hline 23.2 & & & 0.019 & & \\
\hline 24 & & 0.212 & & 0.058 & 0.091 \\
\hline 24.2 & & & 0.019 & & \\
\hline 25 & & 0.135 & & 0.043 & 0.029 \\
\hline 25.2 & & & 0.005 & & \\
\hline 26 & & 0.067 & & 0.005 & 0.010 \\
\hline 26.2 & & & 0.063 & & \\
\hline 27 & 0.005 & 0.010 & & 0.010 & \\
\hline 27.2 & & & 0.043 & & \\
\hline 28 & 0.111 & 0.010 & & & \\
\hline 28.2 & & & 0.038 & & \\
\hline 29 & 0.284 & 0.005 & & & \\
\hline 29.2 & & & 0.058 & & \\
\hline 30 & 0.322 & & & & \\
\hline 30.2 & 0.014 & & 0.043 & & \\
\hline 31 & 0.043 & & & & \\
\hline 31.2 & 0.048 & & 0.048 & & \\
\hline 32 & 0.014 & & & & \\
\hline 32.2 & 0.111 & & 0.072 & & \\
\hline 33 & & & 0.005 & & \\
\hline 33.2 & 0.038 & & 0.010 & & \\
\hline \multicolumn{6}{|l|}{34} \\
\hline 34.2 & 0.005 & & 0.005 & & \\
\hline 35 & 0.005 & & & & \\
\hline \multicolumn{6}{|l|}{35.2} \\
\hline \multicolumn{6}{|l|}{36} \\
\hline \multicolumn{6}{|l|}{36.2} \\
\hline 37 & & & & & \\
\hline
\end{tabular}

\begin{tabular}{|c|c|c|c|c|c|c|c|c|c|c|c|c|c|c|c|c|c|c|c|c|c|}
\hline & D3S1358 & $\mathrm{vWA}$ & D16S539 & CSFIPO & TPOX & D8S1179 & D21S11 & D18S51 & D2S441 & D19S433 & TH01 & FGA & D22S1045 & D5S818 & D13S317 & D7S820 & SE33 & D10S1248 & D1S1656 & D12S391 & D2S1338 \\
\hline MP & 0.123 & 0.086 & 0.093 & 0.164 & 0.211 & 0.055 & 0.082 & 0.042 & 0.096 & 0.048 & 0.087 & 0.040 & 0.193 & 0.077 & 0.073 & 0.092 & 0.015 & 0.121 & 0.048 & 0.035 & 0.047 \\
\hline PD & 0.877 & 0.914 & 0.907 & 0.836 & 0.789 & 0.945 & 0.918 & 0.958 & 0.904 & 0.952 & 0.913 & 0.960 & 0.807 & 0.923 & 0.927 & 0.908 & 0.985 & 0.879 & 0.952 & 0.965 & 0.953 \\
\hline PIC & 0.681 & 0.745 & 0.740 & 0.626 & 0.551 & 0.815 & 0.757 & 0.835 & 0.724 & 0.830 & 0.743 & 0.844 & 0.573 & 0.757 & 0.779 & 0.737 & 0.939 & 0.696 & 0.826 & 0.862 & 0.831 \\
\hline PE & 0.347 & 0.416 & 0.402 & 0.416 & 0.275 & 0.650 & 0.493 & 0.613 & 0.446 & 0.706 & 0.510 & 0.687 & 0.253 & 0.578 & 0.526 & 0.416 & 0.823 & 0.578 & 0.687 & 0.632 & 0.493 \\
\hline TPI & 1.405 & 1.625 & 1.576 & 1.625 & 1.209 & 2.889 & 1.926 & 2.600 & 1.733 & 3.467 & 2.000 & 3.250 & 1.156 & 2.364 & 2.080 & 1.625 & 5.778 & 2.364 & 3.250 & 2.737 & 1.926 \\
\hline Но & 0.356 & 0.308 & 0.317 & 0.308 & 0.413 & 0.173 & 0.260 & 0.192 & 0.288 & 0.144 & 0.250 & 0.154 & 0.433 & 0.212 & 0.240 & 0.308 & 0.087 & 0.212 & 0.154 & 0.183 & 0.260 \\
\hline
\end{tabular}




\begin{tabular}{|c|c|c|c|c|c|c|c|c|c|c|c|c|c|c|c|c|c|c|c|c|c|}
\hline $\mathrm{Ht}$ & 0.644 & 0.692 & 0.683 & 0.692 & 0.587 & 0.827 & 0.740 & 0.808 & 0.712 & 0.856 & 0.750 & 0.846 & 0.567 & 0.788 & 0.760 & 0.692 & 0.913 & 0.788 & 0.846 & 0.817 & 0.740 \\
\hline les & 208 & 208 & 208 & 208 & 208 & 208 & 208 & 208 & 208 & 208 & 208 & 208 & 208 & 208 & 208 & 208 & 208 & 208 & 208 & 208 & 208 \\
\hline
\end{tabular}

MP: Random Match Probability, PD: Power of Discrimination, PIC: Polymorphism information content, PE: Power of Exclusion, TPI: Typical Paternity Index, Ho: Homozygosity, Ht: Heterozygosity 
Supplementary material, Table S1d. Allele frequencies and forensic statistics (Southern region)

\begin{tabular}{|c|c|c|c|c|c|c|c|c|c|c|c|c|c|c|c|c|c|c|c|c|c|}
\hline Allele & D3S1358 & vWA & D16S539 & CSFIPO & TPOX & D8S1179 & D21S11 & D18S51 & $\mathrm{D} 2 \mathrm{~S} 441$ & D19S433 & TH01 & FGA & D22S1045 & D5S818 & D13S317 & D7S820 & SE 33 & D10S1248 & D1S1656 & D12S391 & D2S1338 \\
\hline 4 & & & & & & & & & & & 0.005 & & & & & & & & & & \\
\hline 6 & & & & & & & & & & & 0.262 & & & & & & & & & & \\
\hline 6.3 & & & & & & & & & & & & & & & & & 0.005 & & & & \\
\hline 7 & & & & & 0.005 & & & & & & 0.189 & & & & & 0.034 & & & & & \\
\hline 7.3 & & & & & & & & & & & & & & & & & 0.015 & & & & \\
\hline 8 & & & 0.044 & & 0.534 & 0.005 & & & & & 0.097 & & & 0.015 & 0.155 & 0.121 & & & & & \\
\hline 9 & & & 0.146 & 0.015 & 0.194 & 0.005 & & & 0.019 & & 0.296 & & & 0.058 & 0.024 & 0.058 & & 0.019 & & & \\
\hline 9.3 & & & & & & & & & & & 0.141 & & & & & & & & & & \\
\hline 10 & & & 0.141 & 0.320 & 0.126 & 0.073 & & & 0.107 & & 0.010 & & & 0.160 & 0.049 & 0.383 & 0.005 & & & & \\
\hline 11 & & & 0.320 & 0.359 & 0.136 & 0.146 & & 0.024 & 0.335 & 0.010 & & & 0.083 & 0.194 & 0.199 & 0.199 & 0.005 & 0.015 & 0.029 & & \\
\hline \multicolumn{22}{|l|}{11.2} \\
\hline 11.3 & & & & & & & & & 0.078 & & & & & & & & & & & & \\
\hline 12 & & & 0.136 & 0.267 & 0.005 & 0.155 & & 0.126 & 0.112 & 0.083 & & & 0.005 & 0.393 & 0.364 & 0.184 & 0.005 & 0.024 & 0.121 & & \\
\hline 12.2 & & & & & & & & & & 0.010 & & & & & & & & & & & \\
\hline 13 & 0.024 & & 0.170 & 0.039 & & 0.180 & & 0.209 & 0.005 & 0.194 & & & & 0.170 & 0.146 & 0.015 & 0.015 & 0.262 & 0.146 & & \\
\hline 13.2 & & & & & & & & & & 0.078 & & & & & & & & & & & \\
\hline \multicolumn{22}{|l|}{13.3} \\
\hline 14 & 0.053 & 0.034 & 0.044 & & & 0.204 & & 0.146 & 0.301 & 0.204 & & & 0.053 & 0.010 & 0.063 & 0.005 & 0.044 & 0.335 & 0.131 & & 0.010 \\
\hline 14.2 & & & & & & & & & & 0.053 & & & & & & & & & & & \\
\hline 15 & 0.204 & 0.117 & & & & 0.189 & & 0.068 & 0.034 & 0.121 & & & 0.505 & & & & 0.015 & 0.218 & 0.146 & 0.039 & \\
\hline 15.2 & & & & & & & & & & 0.126 & & & & & & & & & & & \\
\hline 15.3 & & & & & & & & & & & & & & & & & & & 0.024 & & \\
\hline 16 & 0.262 & 0.320 & & & & 0.039 & & 0.097 & 0.010 & 0.068 & & & 0.316 & & & & 0.063 & 0.092 & 0.262 & 0.015 & 0.063 \\
\hline 16.2 & & & & & & & & 0.005 & & 0.039 & & & & & & & & & & & \\
\hline 16.3 & & & & & & & & & & & & & & & & & & & 0.068 & & \\
\hline 17 & 0.350 & 0.296 & & & & 0.005 & & 0.107 & & 0.005 & & & 0.039 & & & & 0.087 & 0.034 & 0.049 & 0.131 & 0.248 \\
\hline 17.2 & & & & & & & & & & 0.010 & & & & & & & & & & & \\
\hline 17.3 & & & & & & & & & & & & & & & & & & & 0.010 & & \\
\hline 18 & 0.097 & 0.165 & & & & & & 0.102 & & & & 0.010 & & & & & 0.126 & & & 0.165 & 0.092 \\
\hline 18.3 & & & & & & & & & & & & & & & & & & & 0.015 & 0.010 & \\
\hline 19 & 0.010 & 0.058 & & & & & & 0.073 & & & & 0.073 & & & & & 0.068 & & & 0.136 & 0.150 \\
\hline 19.3 & & & & & & & & & & & & & & & & & & & & 0.005 & \\
\hline 20 & & 0.005 & & & & & & 0.039 & & & & 0.097 & & & & & 0.058 & & & 0.092 & 0.209 \\
\hline 20.2 & & & & & & & & & & & & & & & & & 0.005 & & & & \\
\hline 21 & & 0.005 & & & & & & 0.005 & & & & 0.136 & & & & & 0.019 & & & 0.117 & 0.073 \\
\hline 21.2 & & & & & & & & & & & & 0.005 & & & & & 0.126 & & & & \\
\hline 22 & & & & & & & & & & & & 0.155 & & & & & & & & 0.117 & 0.034 \\
\hline
\end{tabular}




\begin{tabular}{|c|c|c|c|c|c|}
\hline 22.2 & & & 0.010 & & \\
\hline \multicolumn{6}{|l|}{22.3} \\
\hline 23 & & 0.160 & & 0.117 & 0.053 \\
\hline 23.2 & & 0.005 & 0.024 & & \\
\hline 24 & & 0.214 & & 0.029 & 0.029 \\
\hline 24.2 & & & 0.034 & & \\
\hline 25 & & 0.073 & & 0.019 & 0.039 \\
\hline 25.2 & & & 0.019 & & \\
\hline 26 & & 0.063 & & 0.010 & \\
\hline 26.2 & & & 0.015 & & \\
\hline 27 & 0.010 & 0.005 & & & \\
\hline 27.2 & & & 0.049 & & \\
\hline 28 & 0.155 & 0.005 & & & \\
\hline 28.2 & & & 0.034 & & \\
\hline 29 & 0.296 & & & & \\
\hline 29.2 & & & 0.029 & & \\
\hline 30 & 0.257 & & & & \\
\hline 30.2 & 0.015 & & 0.024 & & \\
\hline 31 & 0.039 & & & & \\
\hline 31.2 & 0.078 & & 0.034 & & \\
\hline \multicolumn{6}{|l|}{32} \\
\hline 32.2 & 0.126 & & 0.019 & & \\
\hline 33 & & & 0.010 & & \\
\hline 33.2 & 0.019 & & 0.010 & & \\
\hline 34 & & & 0.024 & & \\
\hline \multicolumn{6}{|l|}{34.2} \\
\hline \multicolumn{6}{|l|}{35} \\
\hline 35.2 & & & 0.005 & & \\
\hline \multicolumn{6}{|l|}{36} \\
\hline \multicolumn{6}{|l|}{36.2} \\
\hline 37 & 0.005 & & & & \\
\hline
\end{tabular}

\begin{tabular}{|c|c|c|c|c|c|c|c|c|c|c|c|c|c|c|c|c|c|c|c|c|c|}
\hline & D3S1358 & $\mathrm{vWA}$ & D16S539 & CSFIPO & TPOX & D8S1179 & D21S11 & D18S51 & D2S441 & D19S433 & TH01 & FGA & D22S1045 & D5S818 & D13S317 & D7S820 & SE33 & D10S1248 & D1S1656 & D12S391 & D2S1338 \\
\hline MP & 0.102 & 0.099 & 0.072 & 0.149 & 0.177 & 0.054 & 0.069 & 0.032 & 0.088 & 0.037 & 0.094 & 0.046 & 0.181 & 0.108 & 0.079 & 0.095 & 0.015 & 0.106 & 0.043 & 0.030 & 0.049 \\
\hline PD & 0.898 & 0.901 & 0.928 & 0.851 & 0.823 & 0.946 & 0.931 & 0.968 & 0.912 & 0.963 & 0.906 & 0.954 & 0.819 & 0.892 & 0.921 & 0.905 & 0.985 & 0.894 & 0.957 & 0.970 & 0.951 \\
\hline PIC & 0.716 & 0.728 & 0.780 & 0.635 & 0.598 & 0.817 & 0.770 & 0.864 & 0.732 & 0.854 & 0.743 & 0.847 & 0.576 & 0.714 & 0.745 & 0.727 & 0.932 & 0.723 & 0.831 & 0.873 & 0.832 \\
\hline PE & 0.457 & 0.539 & 0.628 & 0.330 & 0.305 & 0.684 & 0.592 & 0.665 & 0.356 & 0.703 & 0.628 & 0.742 & 0.293 & 0.506 & 0.442 & 0.489 & 0.861 & 0.592 & 0.665 & 0.665 & 0.557 \\
\hline TPI & 1.776 & 2.146 & 2.711 & 1.355 & 1.288 & 3.219 & 2.452 & 3.029 & 1.431 & 3.433 & 2.711 & 3.962 & 1.256 & 1.981 & 1.717 & 1.907 & 7.357 & 2.452 & 3.029 & 3.029 & 2.239 \\
\hline Ho & 0.282 & 0.233 & 0.184 & 0.369 & 0.388 & 0.155 & 0.204 & 0.165 & 0.350 & 0.146 & 0.184 & 0.126 & 0.398 & 0.252 & 0.291 & 0.262 & 0.068 & 0.204 & 0.165 & 0.165 & 0.223 \\
\hline
\end{tabular}




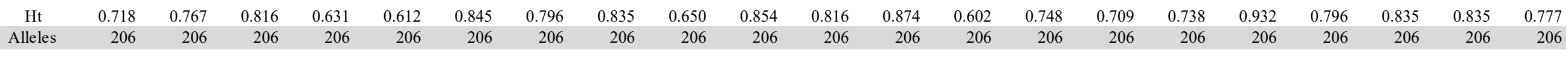

MP: Random Match Probability, PD: Power of Discrimination, PIC: Polymorphism information content, PE: Power of Exclusion, TPI: Typical Paternity Index, Ho: Homozygosity, Ht: Heterozygosity 
Supplementary material, Table S1e. Allele frequencies and forensic statistics (Western region)

\begin{tabular}{|c|c|c|c|c|c|c|c|c|c|c|c|c|c|c|c|c|c|c|c|c|c|}
\hline Allele & D3S1358 & vWA & D16S539 & CSFIPO & TPOX & D8S1179 & $\mathrm{D} 21 \mathrm{~S} 11$ & D18S51 & D2S441 & D19S433 & Тн01 & FGA & D22S1045 & D5S818 & D13S317 & D7S820 & SE33 & D10S1248 & D1S1656 & D12S391 & D2S1338 \\
\hline 4 & & & & & & & & & & & & & & & & & & & & & \\
\hline 6 & & & & & & & & & & & 0.372 & & & & & & & & & & \\
\hline 6.3 & & & & & & & & & & & & & & & & & & & & & \\
\hline 7 & & & & & & & & & & & 0.159 & & & & & 0.004 & & & & & \\
\hline 7.3 & & & & & & & & & & & & & & & & & 0.004 & & & & \\
\hline 8 & & & 0.018 & 0.004 & 0.535 & & & & & & 0.119 & & & 0.009 & 0.097 & 0.199 & & & & & \\
\hline 9 & & & 0.088 & 0.013 & 0.195 & 0.004 & & & 0.009 & & 0.283 & & & 0.058 & 0.022 & 0.115 & 0.004 & 0.031 & & & \\
\hline 9.3 & & & & & & & & & & & 0.066 & & & & & & & & & & \\
\hline 10 & & & 0.133 & 0.283 & 0.119 & 0.022 & & & 0.124 & & & & 0.009 & 0.115 & 0.058 & 0.336 & & & & & \\
\hline 11 & & & 0.376 & 0.292 & 0.137 & 0.119 & & 0.018 & 0.358 & 0.022 & & & 0.124 & 0.257 & 0.252 & 0.155 & & 0.013 & 0.027 & & \\
\hline 11.2 & & & & & & & & & & & & & & & & & 0.004 & & & & \\
\hline 11.3 & & & & & & & & & 0.062 & & & & & & & & & & & & \\
\hline 12 & & & 0.230 & 0.367 & 0.013 & 0.221 & & 0.199 & 0.088 & 0.097 & & & 0.022 & 0.354 & 0.416 & 0.164 & 0.009 & 0.058 & 0.155 & & \\
\hline 12.2 & & & & & & & & & & 0.018 & & & & & & & & & & & \\
\hline 13 & 0.004 & & 0.137 & 0.035 & & 0.212 & & 0.195 & 0.013 & 0.159 & & & & 0.204 & 0.119 & 0.027 & 0.022 & 0.150 & 0.088 & & \\
\hline 13.2 & & & & & & & & & & 0.053 & & & & & & & & & & & \\
\hline 13.3 & & & & & & & & & & & & & & & & & & & & & \\
\hline 14 & 0.066 & 0.027 & 0.018 & 0.004 & & 0.221 & & 0.093 & 0.314 & 0.181 & & & 0.040 & 0.004 & 0.035 & & 0.058 & 0.385 & 0.097 & & 0.004 \\
\hline 14.2 & & & & & & & & & & 0.035 & & & & & & & & & & & \\
\hline 15 & 0.248 & 0.186 & & & & 0.159 & & 0.133 & 0.022 & 0.133 & & & 0.442 & & & & 0.022 & 0.221 & 0.106 & 0.031 & \\
\hline 15.2 & & & & & & & & 0.009 & & 0.159 & & & & & & & & & & & \\
\hline 15.3 & & & & & & & & & & & & & & & & & & & 0.049 & & \\
\hline 16 & 0.314 & 0.292 & & & & 0.031 & & 0.115 & 0.009 & 0.080 & & & 0.296 & & & & 0.062 & 0.080 & 0.261 & 0.018 & 0.080 \\
\hline 16.2 & 0.004 & & & & & & & & & 0.027 & & & & & & & & & & & \\
\hline 16.3 & & & & & & & & & & & & & & & & & & & 0.084 & & \\
\hline 17 & 0.274 & 0.230 & & & & 0.009 & & 0.088 & & 0.004 & & & 0.066 & & & & 0.053 & 0.062 & 0.058 & 0.137 & 0.221 \\
\hline 17.2 & & & & & & & & 0.004 & & 0.031 & & & & & & & & & & & \\
\hline 17.3 & & & & & & & & & & & & & & & & & & & 0.035 & & \\
\hline 18 & 0.084 & 0.199 & & & & & & 0.053 & & & & 0.009 & & & & & 0.106 & & & 0.124 & 0.102 \\
\hline 18.3 & & & & & & & & & & & & & & & & & & & 0.035 & & \\
\hline 19 & 0.004 & 0.044 & & & & & & 0.058 & & & & 0.071 & & & & & 0.062 & & & 0.097 & 0.146 \\
\hline 19.3 & & & & & & & & & & & & & & & & & & & 0.004 & 0.004 & \\
\hline 20 & & 0.022 & & & & & & 0.004 & & & & 0.066 & & & & & 0.031 & & & 0.093 & 0.221 \\
\hline 20.2 & & & & & & & & & & & & & & & & & 0.013 & & & & \\
\hline 21 & & & & & & & & 0.009 & & & & 0.155 & & & & & 0.022 & & & 0.062 & 0.049 \\
\hline 21.2 & & & & & & & & & & & & & & & & & 0.088 & & & & \\
\hline 22 & & & & & & & & 0.018 & & & & 0.097 & & & & & & & & 0.133 & 0.022 \\
\hline
\end{tabular}




\begin{tabular}{|c|c|c|c|c|c|}
\hline 22.2 & & 0.004 & 0.013 & & \\
\hline \multicolumn{6}{|l|}{22.3} \\
\hline 23 & 0.004 & 0.186 & & 0.181 & 0.075 \\
\hline 23.2 & & & 0.013 & & \\
\hline 24 & & 0.235 & & 0.058 & 0.049 \\
\hline 24.2 & & & 0.035 & & \\
\hline 25 & & 0.102 & & 0.053 & 0.027 \\
\hline 25.2 & & & 0.035 & & \\
\hline 26 & & 0.049 & & 0.009 & 0.004 \\
\hline 26.2 & & & 0.066 & & \\
\hline 27 & 0.013 & 0.009 & & & \\
\hline 27.2 & & & 0.058 & & \\
\hline 28 & 0.155 & 0.004 & & & \\
\hline 28.2 & & & 0.058 & & \\
\hline 29 & 0.208 & 0.004 & & & \\
\hline 29.2 & & & 0.013 & & \\
\hline 30 & 0.239 & 0.004 & & & \\
\hline 30.2 & 0.013 & & 0.049 & & \\
\hline 31 & 0.058 & & 0.004 & & \\
\hline 31.2 & 0.115 & 0.004 & 0.058 & & \\
\hline 32 & 0.009 & & & & \\
\hline 32.2 & 0.111 & & 0.031 & & \\
\hline \multicolumn{6}{|l|}{33} \\
\hline 33.2 & 0.053 & & & & \\
\hline \multicolumn{6}{|l|}{34} \\
\hline 34.2 & 0.009 & & 0.004 & & \\
\hline 35 & 0.009 & & & & \\
\hline 35.2 & 0.004 & & & & \\
\hline 36 & 0.004 & & & & \\
\hline \multicolumn{6}{|l|}{36.2} \\
\hline 37 & & & & & \\
\hline
\end{tabular}

\begin{tabular}{|c|c|c|c|c|c|c|c|c|c|c|c|c|c|c|c|c|c|c|c|c|c|}
\hline & D3S1358 & $\mathrm{vWA}$ & D16S539 & CSFIPO & TPOX & D8S1179 & D21S11 & D18S51 & D2S441 & D19S433 & TH01 & FGA & D22S1045 & D5S818 & D13S317 & D7S820 & SE33 & D10S1248 & D1S1656 & D12S391 & D2S1338 \\
\hline MP & 0.104 & 0.079 & 0.092 & 0.144 & 0.171 & 0.066 & 0.043 & 0.039 & 0.107 & 0.032 & 0.109 & 0.046 & 0.143 & 0.102 & 0.118 & 0.084 & 0.014 & 0.093 & 0.037 & 0.033 & 0.041 \\
\hline PD & 0.896 & 0.921 & 0.908 & 0.856 & 0.829 & 0.934 & 0.957 & 0.961 & 0.893 & 0.968 & 0.891 & 0.954 & 0.857 & 0.898 & 0.882 & 0.916 & 0.986 & 0.907 & 0.963 & 0.967 & 0.959 \\
\hline PIC & 0.711 & 0.751 & 0.727 & 0.639 & 0.598 & 0.790 & 0.825 & 0.854 & 0.707 & 0.864 & 0.695 & 0.839 & 0.647 & 0.711 & 0.698 & 0.751 & 0.941 & 0.736 & 0.850 & 0.875 & 0.836 \\
\hline PE & 0.484 & 0.455 & 0.498 & 0.338 & 0.262 & 0.642 & 0.592 & 0.712 & 0.441 & 0.592 & 0.455 & 0.642 & 0.282 & 0.484 & 0.400 & 0.544 & 0.801 & 0.529 & 0.544 & 0.694 & 0.642 \\
\hline TPI & 1.883 & 1.766 & 1.948 & 1.378 & 1.177 & 2.825 & 2.457 & 3.531 & 1.712 & 2.457 & 1.766 & 2.825 & 1.228 & 1.883 & 1.569 & 2.173 & 5.136 & 2.093 & 2.173 & 3.324 & 2.825 \\
\hline Но & 0.265 & 0.283 & 0.257 & 0.363 & 0.425 & 0.177 & 0.204 & 0.142 & 0.292 & 0.204 & 0.283 & 0.177 & 0.407 & 0.265 & 0.319 & 0.230 & 0.097 & 0.239 & 0.230 & 0.150 & 0.177 \\
\hline
\end{tabular}




\begin{tabular}{|c|c|c|c|c|c|c|c|c|c|c|c|c|c|c|c|c|c|c|c|c|c|}
\hline $\mathrm{Ht}$ & 0.735 & 0.717 & 0.743 & 0.637 & 0.575 & 0.823 & 0.796 & 0.858 & 0.708 & 0.796 & 0.717 & 0.823 & 0.593 & 0.735 & 0.681 & 0.770 & 0.903 & 0.761 & 0.770 & 0.850 & 0.823 \\
\hline lleles & 226 & 226 & 226 & 226 & 226 & 226 & 226 & 226 & 226 & 226 & 226 & 226 & 226 & 226 & 226 & 226 & 226 & 226 & 226 & 226 & 226 \\
\hline
\end{tabular}

MP: Random Match Probability, PD: Power of Discrimination, PIC: Polymorphism information content, PE: Power of Exclusion, TPI: Typical Paternity Index, Ho: Homozygosity, Ht: Heterozygosity 
Table S2: Per-locus observed and expected heterozygosity values.

Central SA

\begin{tabular}{lccccc}
\hline Locus & Genotype & alleles & O-Het & E-Het & P-value \\
\hline D3S1358 & 230 & 7 & 0.774 & 0.770 & 0.051 \\
vWA & 230 & 7 & 0.730 & 0.784 & 0.119 \\
D16S539 & 230 & 9 & 0.713 & 0.753 & 0.079 \\
CSF1PO & 230 & 6 & 0.713 & 0.694 & 0.313 \\
TPOX & 230 & 5 & 0.678 & 0.663 & 0.074 \\
D8S1179 & 230 & 9 & 0.826 & 0.829 & 0.609 \\
D21S11 & 230 & 11 & 0.800 & 0.820 & 0.832 \\
D18S51 & 230 & 15 & 0.843 & 0.871 & 0.093 \\
D2S441 & 230 & 8 & 0.765 & 0.753 & $\mathbf{0 . 0 1 2}$ \\
D19S433 & 230 & 14 & 0.809 & 0.880 & 0.492 \\
TH01 & 230 & 6 & 0.652 & 0.725 & 0.239 \\
FGA & 230 & 14 & 0.826 & 0.839 & 0.694 \\
D22S1045 & 230 & 7 & 0.626 & 0.657 & $\mathbf{0 . 0 3 9}$ \\
D5S818 & 230 & 7 & 0.652 & 0.738 & 0.152 \\
D13S317 & 230 & 8 & 0.661 & 0.770 & $\mathbf{0 . 0 3 1}$ \\
D7S820 & 230 & 7 & 0.704 & 0.769 & 0.099 \\
SE33 & 230 & 32 & 0.939 & 0.950 & 0.338 \\
D10S1248 & 230 & 7 & 0.696 & 0.749 & 0.703 \\
D1S1656 & 230 & 14 & 0.861 & 0.873 & 0.759 \\
D12S391 & 230 & 15 & 0.809 & 0.890 & 0.720 \\
D2S1338 & 230 & 13 & 0.783 & 0.834 & 0.108
\end{tabular}

Eastern SA

\begin{tabular}{lccccc}
\hline Locus & Genotype & alleles & O-Het & E-Het & P-value \\
\hline D3S1358 & 176 & 6 & 0.773 & 0.767 & 0.997 \\
vWA & 176 & 8 & 0.727 & 0.793 & 0.053 \\
D16S539 & 176 & 6 & 0.739 & 0.783 & 0.873 \\
CSF1PO & 176 & 7 & 0.682 & 0.720 & 0.733 \\
TPOX & 176 & 6 & 0.511 & 0.602 & 0.365 \\
D8S1179 & 176 & 10 & 0.795 & 0.830 & 0.811 \\
D21S11 & 176 & 11 & 0.750 & 0.819 & 0.473 \\
D18S51 & 176 & 16 & 0.886 & 0.891 & 0.823 \\
D2S441 & 176 & 8 & 0.705 & 0.739 & $\mathbf{0 . 0 2 9}$ \\
D19S433 & 176 & 12 & 0.841 & 0.867 & 0.645 \\
TH01 & 176 & 6 & 0.761 & 0.763 & 0.464 \\
FGA & 176 & 13 & 0.784 & 0.856 & 0.269 \\
D22S1045 & 176 & 6 & 0.614 & 0.668 & 0.412 \\
D5S818 & 176 & 8 & 0.682 & 0.747 & 0.240 \\
D13S317 & 176 & 7 & 0.784 & 0.792 & 0.875 \\
D7S820 & 176 & 7 & 0.795 & 0.771 & 0.313 \\
SE33 & 176 & 26 & 0.898 & 0.954 & 0.099 \\
D10S1248 & 176 & 7 & 0.898 & 0.762 & 0.335 \\
D1S1656 & 176 & 11 & 0.773 & 0.879 & $\mathbf{0 . 0 2 2}$ \\
D12S391 & 176 & 14 & 0.852 & 0.896 & 0.723 \\
D2S1338 & 176 & 10 & 0.795 & 0.855 & 0.081
\end{tabular}

Northern SA

\begin{tabular}{ccccc}
\hline Genotype & alleles & O-Het & E-Het & P-value \\
\hline 208 & 7 & 0.644 & 0.728 & 0.077 \\
208 & 7 & 0.692 & 0.782 & $\mathbf{0 . 0 4 9}$ \\
208 & 8 & 0.683 & 0.776 & $\mathbf{0 . 0 2 1}$ \\
208 & 7 & 0.692 & 0.692 & 0.873 \\
\hline 208 & 5 & 0.587 & 0.594 & 0.757 \\
208 & 9 & 0.827 & 0.840 & 0.217 \\
208 & 12 & 0.740 & 0.789 & 0.163 \\
208 & 14 & 0.808 & 0.855 & 0.663 \\
208 & 10 & 0.712 & 0.763 & 0.556 \\
208 & 11 & 0.856 & 0.851 & 0.577 \\
208 & 7 & 0.750 & 0.781 & 0.553 \\
208 & 12 & 0.846 & 0.864 & 0.927 \\
208 & 6 & 0.567 & 0.620 & 0.627 \\
208 & 7 & 0.788 & 0.792 & 0.947 \\
208 & 8 & 0.760 & 0.809 & 0.250 \\
208 & 7 & 0.692 & 0.775 & $\mathbf{0 . 0 2 8}$ \\
208 & 30 & 0.913 & 0.947 & 0.312 \\
\hline 208 & 8 & 0.788 & 0.741 & 0.556 \\
\hline 208 & 15 & 0.846 & 0.846 & 0.506 \\
\hline 208 & 14 & 0.817 & 0.878 & 0.124 \\
\hline 208 & 10 & 0.740 & 0.852 & $\mathbf{0 . 0 0 7}$
\end{tabular}

Southern SA

\begin{tabular}{ccccc}
\hline Genotype & alleles & O-Het & E-Het & P-value \\
\hline 206 & 7 & 0.718 & 0.758 & 0.410 \\
\hline 206 & 8 & 0.767 & 0.768 & 0.195 \\
\hline 206 & 7 & 0.816 & 0.809 & 0.199 \\
\hline 206 & 5 & 0.631 & 0.699 & 0.192 \\
\hline 206 & 6 & 0.612 & 0.646 & 0.380 \\
206 & 10 & 0.845 & 0.842 & 0.433 \\
\hline 206 & 10 & 0.796 & 0.802 & 0.925 \\
\hline 206 & 12 & 0.835 & 0.880 & 0.733 \\
\hline 206 & 9 & 0.650 & 0.769 & $\mathbf{0 . 0 3 3}$ \\
\hline 206 & 13 & 0.854 & 0.872 & 0.528 \\
\hline 206 & 7 & 0.816 & 0.782 & 0.882 \\
\hline 206 & 13 & 0.874 & 0.866 & 0.072 \\
\hline 206 & 6 & 0.602 & 0.637 & 0.729 \\
\hline 206 & 7 & 0.748 & 0.753 & 0.089 \\
\hline 206 & 7 & 0.709 & 0.779 & 0.614 \\
\hline 206 & 8 & 0.738 & 0.763 & 0.700 \\
\hline 206 & 31 & 0.932 & 0.940 & 0.763 \\
\hline 206 & 8 & 0.796 & 0.764 & 0.390 \\
\hline 206 & 11 & 0.835 & 0.852 & 0.955 \\
\hline 206 & 14 & 0.835 & 0.889 & 0.719 \\
\hline 206 & 11 & 0.777 & 0.852 & $\mathbf{0 . 0 3 1}$ \\
\hline
\end{tabular}


Western SA

\begin{tabular}{lccccc}
\hline Locus & Genotype & alleles & O-Het & E-Het & P-value \\
\hline D3S1358 & 226 & 8 & 0.735 & 0.756 & 0.153 \\
vWA & 226 & 7 & 0.717 & 0.788 & 0.511 \\
D16S539 & 226 & 7 & 0.743 & 0.764 & 0.816 \\
CSF1PO & 226 & 7 & 0.637 & 0.701 & 0.385 \\
TPOX & 226 & 5 & 0.575 & 0.645 & 0.210 \\
D8S1179 & 226 & 9 & 0.823 & 0.819 & 0.684 \\
D21S11 & 226 & 14 & 0.796 & 0.847 & 0.784 \\
D18S51 & 226 & 15 & 0.858 & 0.872 & 0.152 \\
D2S441 & 226 & 9 & 0.708 & 0.748 & 0.490 \\
D19S433 & 226 & 13 & 0.796 & 0.880 & 0.161 \\
TH01 & 226 & 5 & 0.717 & 0.741 & 0.968 \\
FGA & 226 & 15 & 0.823 & 0.858 & 0.079 \\
D22S1045 & 226 & 7 & 0.593 & 0.698 & $\mathbf{0 . 0 2 2}$ \\
D5S818 & 226 & 7 & 0.735 & 0.754 & 0.805 \\
D13S317 & 226 & 7 & 0.681 & 0.738 & $\mathbf{0 . 0 1 0}$ \\
D7S820 & 226 & 7 & 0.770 & 0.786 & 0.293 \\
SE33 & 226 & 28 & 0.903 & 0.948 & 0.182 \\
D10S1248 & 226 & 8 & 0.761 & 0.769 & 0.117 \\
D1S1656 & 226 & 12 & 0.770 & 0.867 & 0.088 \\
D12S391 & 226 & 13 & 0.850 & 0.890 & 0.120 \\
D2S1338 & 226 & 12 & 0.823 & 0.856 & 0.377
\end{tabular}

O-het: observed heterozygosity; E-het: expected heterozygosity; P-values in bold are significant at $<0.05$. 


\begin{tabular}{|c|c|c|c|c|c|c|c|c|c|c|c|c|c|c|c|c|c|c|c|c|c|c|c|c|}
\hline the & & & & & & & & & & & & & & & & & & & & & & & & \\
\hline $\begin{array}{c}\text { locus } \\
\text { CSF1PO }\end{array}$ & $\begin{array}{c}c \\
0.68675\end{array}$ & $\begin{array}{c}\mathbf{E} \\
0.3375\end{array}$ & $\begin{array}{c}\mathrm{N} \\
0.78628\end{array}$ & $\begin{array}{c}\mathbf{s} \\
0.329\end{array}$ & $\begin{array}{c}\mathbf{w} \\
0.9927\end{array}$ & $\begin{array}{c}\text { SA3 } \\
0.00136\end{array}$ & $\begin{array}{c}\text { SA1 } \\
0.18811\end{array}$ & $\begin{array}{c}\text { SA2 } \\
0.61228\end{array}$ & $\begin{array}{c}\text { SA4 } \\
<0.0001\end{array}$ & $\begin{array}{l}\text { BAH } \\
0.00198\end{array}$ & $\begin{array}{c}\text { QAT } \\
0.08727\end{array}$ & $\begin{array}{c}\text { UAE1 } \\
0.0259\end{array}$ & $\begin{array}{c}\text { UAE2 } \\
0.00023\end{array}$ & $\begin{array}{l}\text { KUW } \\
0.57535\end{array}$ & $\begin{array}{c}\text { OMN } \\
0.05938\end{array}$ & $\begin{array}{c}\text { YEM } \\
0.5571\end{array}$ & $\begin{array}{c}\text { JOR } \\
0.01212\end{array}$ & IRQ2 & $\begin{array}{c}\text { EGY2 } \\
000591\end{array}$ & IRN2 & IRQ1 & $\begin{array}{l}\text { IRN1 } \\
\end{array}$ & EGY1 & $\begin{array}{l}\text { locus } \\
\text { CSF1PO }\end{array}$ \\
\hline D10S1248 & 0.61773 & 0.1364 & 0.08436 & 0.13509 & 0.36028 & $\begin{array}{l}0.200350 \\
0.26353\end{array}$ & $\begin{array}{l}1011 \\
\text { N/A }\end{array}$ & $\begin{array}{l}0.0128 \\
\text { N/A }\end{array}$ & $\mathrm{N} / \mathrm{A}$ & N/A & $\mathrm{N} / \mathrm{A}$ & $\begin{array}{l}0.2874 \\
0.2874\end{array}$ & $\begin{array}{l}0.43145 \\
0.4145\end{array}$ & $\mathrm{~N} / \mathrm{A}$ & N/A & $\begin{array}{l}0.53 / \mathrm{A} \\
0\end{array}$ & $<0.0001$ & N/A & N/A & N/A & N/A & N/A & N/A & D10s1248 \\
\hline D12S391 & 0.63325 & 0.80068 & 1843 & 0036 & 069018 & 7 & N/ & N/A & N/ & N/A & N/A & .0001 & $<0.0001$ & N/ & N/A & * & $<0.0001$ & & $N / A$ & N/A & & & & 125391 \\
\hline D135317 & 0.86089 & 88 & & 29 & & & 100 & 2,278 & - & 1931 & 2 & & & 6 & & & & & $<0.0001$ & & & & & 17 \\
\hline D16S & 0.25951 & & & & & & 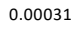 & 4 & & 18 & 00085 & & & 34 & 46 & 11 & 04 & & & & & & & 39 \\
\hline & 0.24444 & & & & & & & & & & & & & & & & & & & & & & & \\
\hline & & & & & & & & & & & & & & & & & & & & & & & & \\
\hline & & & & & & & & & & & & & & & & & & & & & & & & \\
\hline & & & & & & & & & & & & & & & & & & & & & & & & \\
\hline & & & & & & & & & & & & & & & & & & & & & & & & \\
\hline & & & & & & & & & & & & & & & & & & & & & & & & \\
\hline & 0. & & & & & & & & & & & & & & & & & & & & & & & \\
\hline & 0.73 & & & & & & & & & & & & & & & & & & & & & & & \\
\hline & 0.84 & & & & & & & & & & & & & & & & & & & & & & & \\
\hline D851179 & 0.8 & 0.99587 & & 0.01585 & & & & & & $<0.0001$ & & & & & & & 01 & & & & & & & 20 \\
\hline D8S1. & & & & & & & & & & 0.00 & 0.11 & $<0.0001$ & $<0.0001$ & 0.01206 & 0. & 0.06 & $<0.0001$ & & $<0.0$ & & & 01 & & 351179 \\
\hline & & & & & & & & & & & & & & & & & & & & & & & & GA \\
\hline TH & 0.593 & 0. & 0.52141 & 15708 & 0.64356 & 0.32657 & $<0.00$ & 000 & 0.015 & $<0.0$ & 0.627 & 0.00308 & 0.00334 & $<0.0$ & & 0.68552 & $<0.0001$ & & & & & & & THO1 \\
\hline & & 0. & & & & & & 0.832 & & $<0.0$ & & & & & $<0.6$ & & & & & & & & & \\
\hline vWA & 0.68854 & 0.10522 & 0.86834 & 0.56565 & 0.62025 & 0.27522 & 0.00026 & 0.3068 & 0.21439 & $<0.0001$ & 0.00356 & 0.00024 & 0.00068 & 0.51918 & 0.05505 & $<0.0001$ & $<0.0001$ & 0.00007 & 0.00001 & $<0.0001$ & $<0.0001$ & $<0.0001$ & $<0.0001$ & WA \\
\hline
\end{tabular}

\begin{tabular}{|c|c|c|c|c|c|c|c|c|c|c|c|c|c|c|c|c|c|c|c|c|c|c|c|c|}
\hline \multicolumn{25}{|c|}{ ble S3b. Population differentiation exact test, Central subregion. } \\
\hline 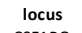 & & & 10 & & & SA3 & JAI & SA2 & SA4 & BAH & QAT & UAE1 & UAE2 & kUW & OMN & . & JOR & 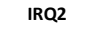 & EGY2 & IRN2 & & & & locu \\
\hline CSF1PO & 0.68675 & 0.22582 & 0.7338 & 0.05758 & 0.77089 & 0.02711 & 0.90817 & 0.28113 & 0.00867 & 0.03938 & 0.04075 & 0.19776 & 0.09154 & 0.74681 & 0.14187 & 0.58021 & 0.20936 & 0.02359 & 0.04761 & 0.10111 & 0.48408 & 0.03193 & 0.08605 & CSF1PO \\
\hline D1051248 & 0.61773 & 0.02465 & 0.56914 & 0.00717 & 0.10645 & 66965 & N/A & N/A & N/A & N/A & 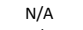 & 13986 & 0.29529 & N/A & $N / A$ & N & $<0.0001$ & $\mathrm{~N} / \mathrm{A}$ & & 14 & & & & D10\$1248 \\
\hline D125391 & 0.63325 & 0.17138 & & & & & $\mathrm{~N} /$ & N/A & 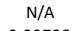 & 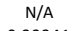 & $\mathrm{N}$ & .0001 & & & & & & & & & & & & \\
\hline D13 & 0.86 & & & & & 3 & 1.01544 & .23255 & .00722 & .33341 & 0.55543 & 17471 & & .21857 & 0.84442 & 52 & 39 & 464 & .00286 & & & 07 & & \\
\hline D1C & 0. & & & & & & & & & & & & & & & & & & & & & & & \\
\hline & & & & & & & & & & & & & & & & & & & & & & & & \\
\hline & & & & & & & & & & & & & & & & & & & & & & & & \\
\hline & & & & & & & & & & & & & & & & & & & & & & & & \\
\hline & & & & & & & & & & & & & & & & & & & & & & & & \\
\hline & & & & & & & & & & & & & & & & & & & & & & & & \\
\hline $\begin{array}{l}\text { D2S13338 } \\
\text { D25441 }\end{array}$ & & $\begin{array}{l}55 \\
35 \\
35\end{array}$ & & & & & & & & $<0.0$ & & & & & & & & & & & & & & \\
\hline 1 & $\begin{array}{l}0 . \\
0 .\end{array}$ & 35 & 99521 & 3 & $\begin{array}{l}68695 \\
57316\end{array}$ & & $\mathrm{~N} /$ & & $\mathrm{N}$ & N/A & $\mathrm{N} / \mathrm{H}$ & 5682 & 0.0 & & & & 515 & & & & & & & \\
\hline & & & & & & & & & & & & & & & 0.3 & & 0.56716 & & & & & & & 358 \\
\hline & & & & & & & & & & & & & & & & & & & & & & & & \\
\hline & & & & & & & & & & & & & & & & & & & & & & & & 179 \\
\hline $\mathrm{FG}$ & & & 0.0 & & 0 & & 0.00301 & 0.32887 & 0.57 & & & 0.0 & 0.0 & 0.18317 & 0.02546 & 0.01308 & 0.00012 & 0.00066 & 0.03351 & $<0.0001$ & 0.0093 & $<0.0001$ & & \\
\hline & & & & & & & & & & & & & & & & & & & & & & & & 33 \\
\hline & & & & & 0.8 & & & & & & & 0.0 & & & $<0.0$ & & 01 & & & & & & & Тн01 \\
\hline & & & & & 57179 & & & 0.6 & & & & & & 0.21 & 0.01 & & 0.09548 & & & & & & & TP \\
\hline VWA & 68854 & 102532 & 0.81971 & 0.45718 & 13949 & 0.51254 & 0.00273 & 0.39756 & 59695 & 0.00276 & 0.08762 & 0.45245 & 0.36965 & 0.60716 & 0.3199 & 0.00146 & 0.04274 & 0.02303 & 0.39773 & D.10779 & 0.36052 & c0.0001 & 0.01838 & WWA \\
\hline
\end{tabular}

K - KSA overall sample, C - Central SA, E - Eastern SA, N - Northern SA, S - Southern SA, W - Western SA (all our data), BAH - Bahrain [1], EGY1, EGY2 - Egypt, respectively [2, 3]; IRN1, IRN2 - Iran, respectively [3, 4]; IRQ1, IRQ2 - Iraq, respectively [3, 5]; JOR - Jordan [6]; KUW - Kuwait [3]; OMN - Oman [7]; QAT - Qatar [8]; SA1, SA2, SA3, SA4 - Saudi Arabia, respectively [3, 7, 9, 10]; UAE1, UAE2 - United Arab Emirates, respectively [11, 12]; YEM - Yemen [7].

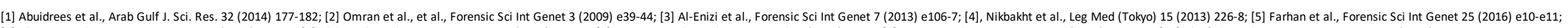
[6] Al-Eitan et al., Aus. J. Forensic Sci. 50 (2016) 97-109; [7] Alshamali et al., Forensic Sci Int 152 (2005) 267-79; [8] Perez-Miranda et al., Hum Hered 61 (2006) 67-79; [9] Osman et al., J. Forensic Res. 6 (2015) 1000267; [10] Alsafiah et al,, Forensic Sci Int Genet 31 (2017) e59-e61; [6] Al-Eitan et al., Aus. J. Forensic Sci. 50 (2016) 97-109; [7] Alshamali et al., Forensic Sci Int 152 (2005) 267-79; [8] Perez-Mir
[11] Jones et al., Forensic Sci Int Genet 28 (2017) e41-e42; [12] Ali Alhmoudi et al., Forensic Sci Int Genet 19 (2015) 190-191. 


\begin{tabular}{|c|c|c|c|c|c|c|c|c|c|c|c|c|c|c|c|c|c|c|c|c|c|c|c|c|}
\hline \multicolumn{25}{|c|}{ be S3c. Population differentiation exact tests, Eastern subregio } \\
\hline locus & $\kappa$ & & & 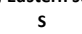 & & SA3 & SA1 & $S A$ & SA4 & BAH & QAT & UAE1 & UAE2 & kUw & OMN & YEM & JOR & IRQ2 & EGY & IRN2 & IRQ1 & RNN1 & EGY1 & loct \\
\hline & 0.68675 & 0.22582 & 0.25975 & 0.12998 & 0.6679 & 0.68821 & 0.09569 & 0.88949 & 0.76175 & 0.58601 & 0.90592 & 0.4 & 0.07181 & 0.71545 & 0.69215 & 0.61136 & 0.81835 & 0.68551 & 0.44157 & 0.03858 & 0.48567 & 0.09057 & 0.82176 & CSFI \\
\hline D10S1248 & 0.61773 & 0.02465 & 0.00039 & 0.12498 & 0.08318 & 0.1823 & N/A & N/A & N/A & N/A & N/A & 0.16433 & 0.08238 & N/A & N/A & $N / A$ & $<0.0001$ & 促 & & $N / 4$ & N/n & N/A & N/A & D10S1248 \\
\hline D12s391 & 0.63325 & 0.17138 & 0.38173 & 30876 & 0.57428 & 0.72899 & N/A & $\mathrm{N} / \mathrm{h}$ & N/A & N/A & N/A & 0.07643 & 0.10129 & N/A & $\mathrm{N} / \mathrm{A}$ & $\mathrm{N} / \mathrm{A}$ & 0.2007 & & $\mathrm{~N}$ & $\mathrm{~N} / \mathrm{A}$ & & N/A & $\mathrm{N} / \mathrm{A}$ & D125391 \\
\hline D1353 & 0.86089 & & & 99 & & 23627 & .03176 & 0.57906 & .17885 & .72209 & 4487 & 03 & & & 0.47063 & .48422 & 0.11708 & & & & 16226 & & 65 & 17 \\
\hline D1655 & 0.25951 & 6 & & & & & & 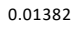 & 17943 & & 0.05484 & & & & & & & & & & & & & 39 \\
\hline & & & & & & & & & & & & & & & & & & & & & & & & \\
\hline & & & & & & & & & & & & & & & & & & & & & & & & \\
\hline & & & & & & & & & & & & & & & & & & & & & & & & \\
\hline & & & & & & & & & & & & & & & & & & & & & & & & \\
\hline & & & & & & & & & & & & & & & & & & & & & & & & \\
\hline & & & & & & & & & & & & & & & & & & & & & & & & \\
\hline & & & & & & & & & & & & & & & & & & & & & & & & \\
\hline & 0. & & & & & & & & & & & & & & & & & & & & & & & \\
\hline & 0.84 & & & & & & & & & & & & & & & & & & & & & & & \\
\hline D75820 & $\begin{array}{l}0.8 \\
0.95\end{array}$ & & & & & & & & & & & & & & & & & & & & & & & 20 \\
\hline 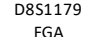 & & & & & & & & & & & & & & & & & & & & & & & & 179 \\
\hline $\begin{array}{l}\mathrm{FG} \\
\mathrm{SEB}\end{array}$ & $\begin{array}{l}0.50292 \\
0.96352\end{array}$ & 0. & 0. & 7 & 11 & & N/A & N/ & $\mathrm{N}$ & $\mathrm{N}$ & $\mathrm{N}$ & & $\begin{array}{l}0.86842 \\
0.95817\end{array}$ & $\mathrm{~N}$ & $\begin{array}{l}0.57 \\
\mathrm{~N} /\end{array}$ & $\mathrm{N}$ & 0.2 & & $N$ & & $\begin{array}{l}.65079 \\
\text { N/A }\end{array}$ & & & A \\
\hline THO & 0. & 0. & 0. & & 0. & 0.31649 & 0.00 & 0.01 & & & & 0. & 0.1 & $<0.0$ & 0.00092 & 0.78 & 0.48487 & & 0.1 & & & & & TH01 \\
\hline TPO & 0 . & & & & & & & 0.531 & 0.35419 & & 0.52 & 0.16 & & 0.08 & 0.00 & & & & & & & & & TP \\
\hline vWA & 68854 & 02532 & 0.06767 & 0.05344 & 0.49898 & 0.01593 & 0.00391 & 37689 & 0.09417 & 00001 & 0.01029 & 0.00031 & 0.00236 & 0.04388 & 0.05682 & .00004 & 0.02966 & 0.02371 & .00049 & 0.0001 & 0.00124 & 0.0001 & .00001 & WA \\
\hline
\end{tabular}

\begin{tabular}{|c|c|c|c|c|c|c|c|c|c|c|c|c|c|c|c|c|c|c|c|c|c|c|c|c|}
\hline & & & & & & SA3 & SA1 & & SA4 & BAH & QAT & UAE1 & UA & KU & OM & YEM & JOR & IRQ2 & EG & $\mathbf{I R}$ & IRQ1 & |RT| & EG & \\
\hline & 0.686 & 0.22582 & 0.25975 & 0.13782 & 0.9667 & 0.04099 & 0.81335 & 0.54205 & 0.0172 & 0.07878 & 0.0899 & 0.29083 & 0.13506 & 0.80722 & 0.07494 & 0.59895 & 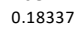 & 0.04431 & 0.26 & 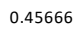 & .29287 & .11901 & 43915 & 0 \\
\hline D1 & 0.61773 & 0.02465 & 0.00039 & 00098 & & & N/A & $\mathrm{N} / \mathrm{A}$ & & $\mathrm{N} / \mathrm{A}$ & N/A & & & & N/A & & & & & & & & & \\
\hline & 0.63325 & 0.17138 & 8173 & 4721 & 0.00379 & & $\mathrm{~N} / \mathrm{A}$ & $N / A$ & & $\mathrm{~N} / \mathrm{A}$ & N/A & & 0.00833 & & $\mathrm{~N} / \mathrm{A}$ & & 0.00308 & & & & & & & 391 \\
\hline D135317 & 0.86089 & 0.96799 & 0.40396 & 00832 & 0.00971 & 00137 & 0.0002 & 0.27553 & 0.00057 & 0.01705 & 0.35944 & 0.00201 & 0.00074 & 0.00947 & 0.03976 & 0.01315 & 0.00142 & 0.0109 & 0.00152 & 0.00167 & 0.00031 & 0.00039 & 0.01245 & D135317 \\
\hline D165539 & 0.25951 & 0.18926 & 19648 & & 0.9707 & 10775 & 0.00341 & 0.0141 & 0.01782 & 0.20111 & 01915 & 0.06082 & 0.02047 & 0.19161 & 0.48892 & 0.20659 & 0.03092 & 0.36479 & 0.03012 & & 0.0112 & 0.3 & & D165539 \\
\hline & 0.24444 & 4791 & 0.04378 & 104 & 0.00319 & 25 & 0.018 & 0.0121 & 025 & & & 0.16037 & & 0.16419 & 0.0237 & 0.41536 & 0.02326 & 0.01261 & $<0.0001$ & & 0.03651 & & & 18551 \\
\hline D1S & 0.4009 & & 122 & & & & 0.15387 & $N$ & & 0.01078 & 0.00087 & 51 & & 0.92544 & & . & & 0.04773 & 0.24022 & 0.28758 & 0.27279 & 0.27104 & 0.47736 & 33 \\
\hline D1S & 0.2 & & & & & & N & & & & & & & & & & & & & & & & & 556 \\
\hline & & & & & & & & & & & & & & & & & & & & & & & & \\
\hline D22S & & & & & & & $\mathrm{N} /$ & $\mathrm{N} / \mathrm{A}$ & $\mathrm{N}$ & $\mathrm{N}$ & $N$ & & & & NA & & & & N & & & N/4 & & \\
\hline D2S1 & 0.3 & & & & & & 0.04 & $\mathrm{~N}$ & 0.1 & $<0.0$ & 0.0 & & & 0.0 & & & & & & $<0$. & & $<0$. & & \\
\hline & & & & & & & & & & & & & & & & & & & & & & & & \\
\hline & & & & & & & & & & & & & & & & & & & & & & & & \\
\hline & 0.8 & & & & & & & & & & & & & & & & & & & & & & & \\
\hline & & & & & & & & & & & & & & & & & & & & & & & & \\
\hline D8S & & & & & & & & & & & & & & & & & & & & & & & & 179 \\
\hline FG & & & & & & & 0.0 & & & & & & & & & & & & & & & & & \\
\hline & & & & & & & & & & & & & & & & & & & & & & & & \\
\hline & & & & & & & & & & & & & & & & & & & & & & & & \\
\hline & & & & & & & & & & & & & & & & & & & & & & & & \\
\hline vWf & 854 & 0.02532 & 0.06767 & 0.6751 & 84 & 35446 & 181 & 752 & 0.80603 & 214 & 0.10503 & 334 & 307 & 11 & 0.18352 & ๔..0001 & 1116 & 0.00995 & .30516 & 5733 & 0.23645 & 0.0001 & 0.04407 & A \\
\hline
\end{tabular}

K - KSA overall sample, C - Central SA, E - Eastern SA, N - Northern SA, S - Southern SA, W - Western SA (all our data), BAH - Bahrain [1], EGY1, EGY2 - Egypt, respectively [2, 3]; IRN1, IRN2 - Iran, respectively [3, 4]; IRQ1, IRQ2 - Iraq, respectively [3, 5]; JOR - Jordan [6]; KUW - Kuwait [3]; OMN - Oman [7]; QAT - Qatar [8]; SA1, SA2, SA3, SA4 - Saudi Arabia, respectively [3, 7, 9, 10]; UAE1, UAE2 - United Arab Emirates, respectively [11, 12]; YEM - Yemen [7].

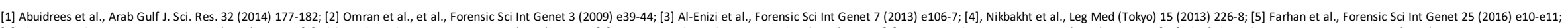
[6] Al-Eitan et al., Aus. J. Forensic Sci. 50 (2016) 97-109; [7] Alshamali et al., Forensic Sci Int 152 (2005) 267-79; [8] Perez-Miranda et al., Hum Hered 61 (2006) 67-79; [9] Osman et al., J. Forensic Res. 6 (2015) 1000267; [10] Alsafiah et al,, Forensic Sci Int Genet 31 (2017) e59-e61; [6] Al-Eitan et al., Aus. J. Forensic Sci. 50 (2016) 97-109; [7] Alshamali et al., Forensic Sci Int 152 (2005) 267-79; [8] Perez-Mir
[11] Jones et al., Forensic Sci Int Genet 28 (2017) e41-e42; [12] Ali Alhmoudi et al., Forensic Sci Int Genet 19 (2015) 190-191. 


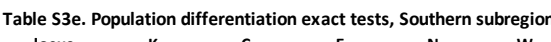

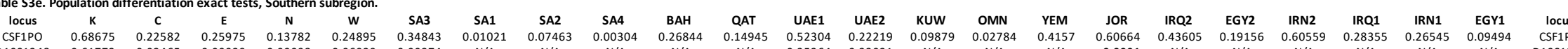
$\begin{array}{llllllllllllllllllllll} & & & \end{array}$

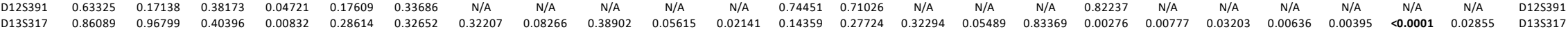

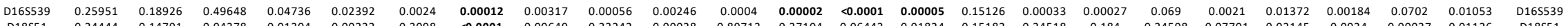

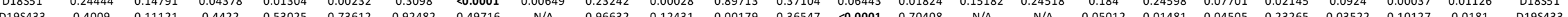

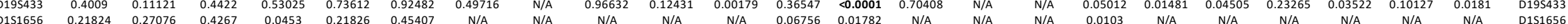
$\begin{array}{lllllllllllllllllllllllllll}\mathrm{D} 21511 & 0.67154 & 0.26485 & 0.07001 & 0.43857 & 0.22524 & 0.88613 & 0.04392 & 0.4274 & 0.16967 & 0.0311 & 0.32994 & 0.57611 & 0.20823 & 0.71905 & 0.04769 & 0.74561 & 0.01775 & 0.23011 & 0.07005 & 0.08368 & 0.6585 & 0.64421 & 0.81566 & 021511\end{array}$

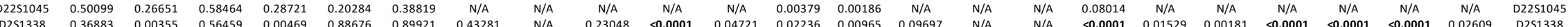

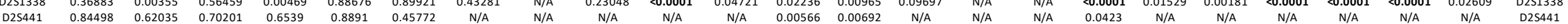

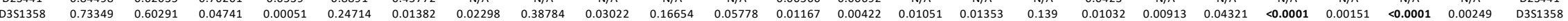
$\begin{array}{lllllllllllllllllllllllll}\mathrm{D} 55818 & 0.84216 & 0.38549 & 0.0158 & 0.00121 & 0.50895 & 0.0142 & 0.1696 & 0.05942 & 0.05923 & 0.41131 & 0.06144 & 0.17181 & 0.29423 & 0.23433 & 0.9758 & 0.92176 & <.0001 & 0.0718 & 0.13151 & 0.01403 & 0.02177 & 0.02522 & 0.13816 & 0.055818\end{array}$ $\begin{array}{lllllllllllllllllllllllll}\mathrm{D} 78820 & 0.815 & 0.88101 & 0.17329 & 0.00086 & 0.00971 & 0.0073 & 0.0373 & 0.11754 & 0.00058 & 0.02937 & 0.02049 & 0.0192 & 0.00774 & 0.00646 & 0.56223 & 0.00816 & 0.00501 & 0.10405 & 0.0157 & 0.00092 & 0.00438 & 0.01236 & 0.0359 & \text { D75820 }\end{array}$

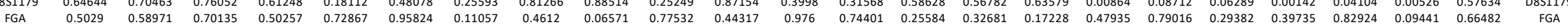

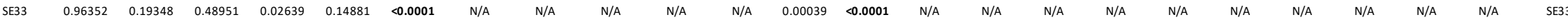
$\begin{array}{lllllllllllllllllllllllllll}\text { TH01 } & 0.59308 & 0.22986 & 0.17408 & 0.57206 & 0.01203 & 0.36805 & 0.00733 & 0.00017 & 0.31227 & 0.05653 & 0.1092 & 0.78628 & 0.42087 & <0.0001 & 0.01096 & 0.39218 & 0.34276 & 0.7518 & 0.09073 & 0.20991 & 0.27839 & 0.16788 & 0.04238 & \text { TH01 }\end{array}$ $\begin{array}{llllllllllllllllllllllll}\text { TPOX } & 0.71056 & 0.14632 & 0.34267 & 0.3771 & 0.94066 & 0.50331 & 0.48816 & 0.50193 & 0.12158 & 0.00409 & 0.01113 & 0.01335 & 0.01626 & 0.01593 & 0.00141 & 0.7826 & 0.00442 & 0.00051 & 0.00377 & <0.0001 & 0.00001 & 0.0009 & 0.01258 \\ \text { vWA } & 0.68854 & 0.02532 & 0.06767 & 0.6751 & 0.18432 & 0.86104 & 0.00879 & 0.36288 & 0.61975 & 0.02889 & 0.03277 & 0.23519 & 0.25382 & 0.30711 & 0.33496 & 0.00039 & 0.1016 & 0.00472 & 0.0313 & 0.04107 & 0.15314 & <0.0001 & 0.20703\end{array}$

Table S3f. Population differentiation exact tests, Western subresion.

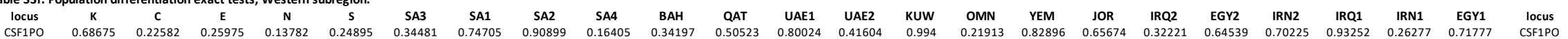

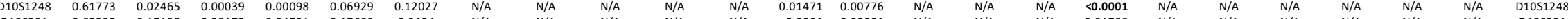

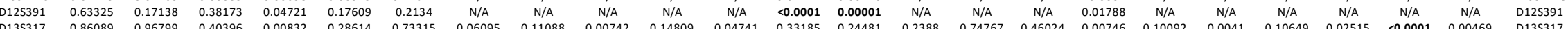

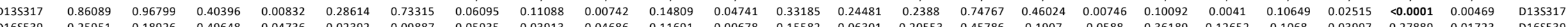
$\begin{array}{lllllllllllllllllllllllllllllllll}D 18551 & 0.24444 & 0.14791 & 0.04378 & 0.01304 & 0.00232 & 0.34796 & 0.0447 & 0.34362 & 0.15895 & 0.00044 & 0.00553 & 0.02304 & 0.00054 & 0.34893 & 0.00416 & 0.44326 & <0.0001 & 0.04178 & 0.0042 & 0.00014 & 0.00022 & 0.00018 & 0.04472 & 018551\end{array}$

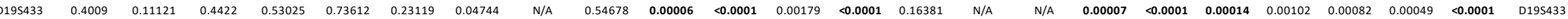

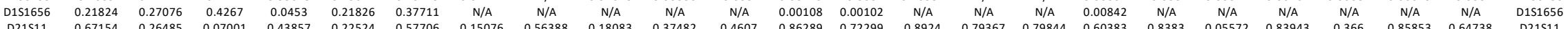

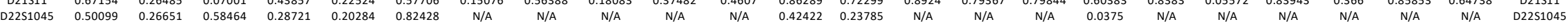

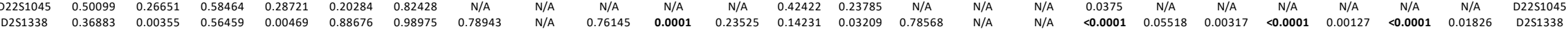

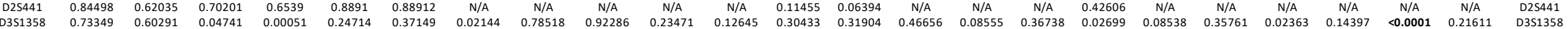

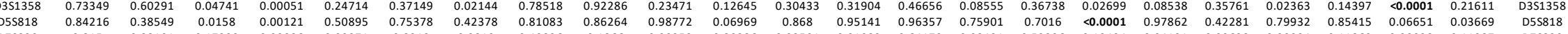

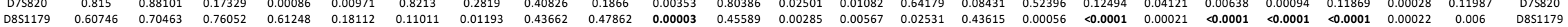

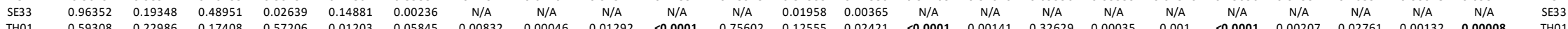

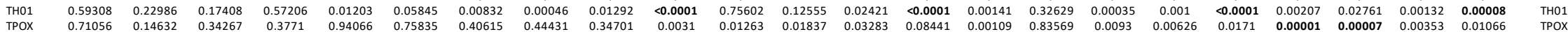

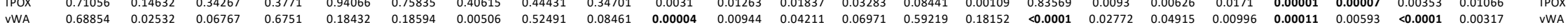

K- KSA overall sample, C - Central SA, E - Eastern SA, N - Northern SA, S - Southern SA, W - Western SA (all our data), BAH - Bahrain [1], EGY1, EGY2 - Egypt, respectively [2, 3]; IRN1, IRN2 - Iran, respectively [3, 4]; IRQ1, IRQ2 - Iraq, respectively [3, 5]; JOR - Jordan [6]; KUW - Kuwait [3]; OMN - Oman [7]; QAT - Qatar [8]; SA1, SA2, SA3, SA4 - Saudi Arabia, respectively [3, 7, 9, 10]; UAE1, UAE2 - United Arab Emirates, respectively [11, 12]; YEM - Yemen [7].

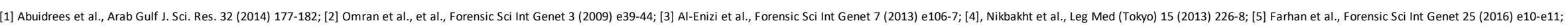
[6] Al-Eitan et al., Aus.J. Forensic Sci. 50 (2016) 97-109; [7] Alshamali et al, Forensic Sci Int 152 (2005) 267-79; [8] Perez-Miranda et al., Hum Hered 61 (2006) 67-79; [9] Osman et al, J. Forensic Res. 6 (2015) 1000267; [10] Alsafiah et al., Forensic Sci Int Genet 31 (2017) e59-e61; [11] Jones et al., Forensic Sci Int Genet 28 (2017) e41-e42; [12] Ali Alhmoudi et al., Forensic Sci Int Genet 19 (2015) 190-191. 